\title{
FreSpeD: Frequency-Specific Change-Point Detection in Epileptic Seizure Multi-Channel EEG Data
}

\author{
Anna Louise Schröder* and Hernando Ombao ${ }^{\dagger}$
}

April 24, 2018

\begin{abstract}
The goal in this paper is to develop a practical tool that identifies changes in the brain activity as recorded in electroencephalograms (EEG). Our method is devised to detect possibly subtle disruptions in normal brain functioning that precede the onset of an epileptic seizure. Moreover, it is able to capture the evolution of seizure spread from one region (or channel) to another. The proposed frequency-specific change-point detection method (FreSpeD) deploys a cumulative sum-type test statistic within a binary segmentation algorithm. We demonstrate the theoretical properties of FreSpeD and show its robustness to parameter choice and advantages against two competing methods. Furthermore, the FreSpeD method produces directly interpretable output. When applied to epileptic seizure EEG data, FreSpeD identifies the correct brain region as the focal point of seizure and the timing of the seizure onset. Moreover, FreSpeD detects changes in cross-coherence immediately before seizure onset which indicate an evolution leading up to the seizure. These changes
\end{abstract}

*Department of Statistics, London School of Economics, London WC2A 2AE, UK; almschroeder@alumni.lse.ac.uk

${ }^{\dagger}$ Statistics Program, King Abdullah University of Science an Technology, Saudi Arabia; hernando.ombao@kaust. edu.sa 
are subtle and were not captured by the methods that previously analyzed the same EEG data.

Keywords: Change Points; Coherence Analysis; CUSUM; Electroencephalograms; Epileptic Seizure; Multivariate Time Series; Spectral Analysis.

\section{Introduction}

Epileptic seizures have received much attention by the neuroscience community. The analysis of these episodes of abnormal brain activity helps researchers understand disruptions in normal brain functioning with the ultimate goal of developing more precise diagnosis, improved therapy and effective early-warning systems for onset of seizure activity. We analyze here electroencephalographic (EEG) recording of a spontaneous (non-induced) seizure and focus on the temporal evolution of energy distributions at individual EEG channels and interactions between channel pairs. We develop a new statistical tool that characterizes the seizure and evaluate its evolution over both space (across channels) and time. Guided by the fact that EEGs can be decomposed into waveforms with different oscillations (ranging from low to high frequencies) our proposed method identifies pre-seizure build up of subtle changes in the cross-coherence between channels. These changes have not been identified by other methods and cannot be detected by visual inspection. Our method offers new insights to the temporal and spatial location of abrupt changes in the energy spectrum during the seizure, with the feature of identifying the specific frequency bands that drive these changes.

During seizure the spectral profile of brain activity displays sudden and abrupt changes. To capture this in a parsimonious but effective way, EEG recordings are frequently modelled as piecewise stationary process (see e.g. Saab and Gotman, 2005; Terrien et al., 2013; Kumar et al., 2014). As a direct implication, a multivariate change-point detection method is required to identify change points and thus produce a segmentation of the multi-channel EEG data into quasi-stationary blocks. The method introduced here allows not only this segmentation, but also direct inter- 
pretation in the sense of assigning a change point to one or multiple EEG channels and frequency bands. In this work, we will use the terms change-point detection and segmentation interchangeably.

Much effort has been dedicated in the past to developing segmentation methods that rely on detection of change points in model parameters, see for example, the (vector) autoregressive ((V)AR) models in Kirch et al. (2015), Davis et al. (2006), Chen et al. (2010) and the works referenced in Chen and Gupta (2012). Parametric change-point detection methods are efficient if the underlying model is correct, but one can expect that possible model misspecification severely affects the performance. Another limitation of the parametric approach is the difficulty in the interpretation of detecting a change in a parameter. For example, a change in a high-order lag parameter of a VAR model is not straight-forward to explain to a neuroscientist or clinician. On the contrary, most neuroscientists have a better intuition for the conventional approach of monitoring the energy distribution over different oscillations in the Fourier domain.

Another class of change-point methods are nonparametric and focus on detecting changes in the spectral characteristics of EEG data. See, for example, Adak (1998), Ombao et al. (2005), Terrien et al. (2013) and references cited therein. One feature common to many of these methods is the use of a dyadic segmentation which restricts both the permitted data length and the change-point locations to having dyadic power $2^{i}, i$ some positive integer. Another shared feature is that change detection lacks specificity in frequency which is a serious limitation because, for interpretability, methods for EEG seizure must be able to identify not only the change points but also the frequency bands to which these changes can be attributed. Such frequency-specific change detection can help to link the segmentation results to neuroscientific findings on event-related changes in brain activity at different frequency bands (Alarcon et al., 1995; Blondin and Greer, 2011; Schmitt et al., 2012).

Our contribution in this paper is a method that identifies frequency band-specific temporal changes in both the spectral energy distribution at each channel and in the cross-coherence between pairs of channels. Consequently, our proposed method is able to capture the evolution of seizure spread from one region (or channel) to 
another. The detection of frequency-specific (or frequency band-specific) changes for a subset of frequency bands has been discussed in the literature, e.g. by Kaplan et al. (2001) and Saab and Gotman (2005). However, detection of frequency-specific change points in coherence has received limited attention to date. Coherence is a frequency-specific measure of linear relation between a pair of channels. More formally, coherence at one frequency band is asymptotically equivalent to the square of the linear correlation between this frequency band's oscillations (obtained by filtering) at a pair of channels, as shown in Ombao and van Bellegem (2008). The evolution of coherence between EEG activity at different channels or neuronal activity between regions on the cortical surface can provide interesting insights. For example, Sun et al. (2004) showed time-invariant differences in coherence for different motor tasks. In a learning experiment, where a subject determines associations between two sets of pictures, Gorrostieta et al. (2012) showed that coherence between the nucleus acumbens and the hippocampus evolves during the experiment. More generally, the interactions between brain regions is increasingly receiving attention in biostatistics and neuroscience. A good example are recent works on the detection of temporal change points in functional magnetic resonance imaging (fMRI, see e.g. Aston et al., 2012; Cribben et al., 2012, 2013). However, fMRI data generally is collected at much lower temporal resolution and therefore inherently cannot be used to analyze the fast-progressing evolution of events like epileptic seizure.

To detect the number and location of possibly many change points of the piecewisestationary EEG process we deploy a multivariate cumulative sum (CUSUM)-type procedure that consistently detects change points in autospectra and coherences of the epileptic seizure EEG data. To give an intuition, the central test statistic identifies at any stage of the iterative algorithm the point that gives the maximum aggregated contrast between adjoining blocks in a given interval, over a set of time series. If the test statistic exceeds a theoretically derived threshold, for which we provide also practical guidance here, we save the thus detected change point and proceed on evaluating the two subintervals resulting from dividing the original interval at this change point. The proposed procedure is motivated by the Sparsified Binary Segmentation algorithm (Cho and Fryzlewicz, 2015a), a generic multiple change-point 
detection methodology that the authors apply to a multivariate locally stationary wavelet process to detect change points in Haar wavelet periodograms and crossperiodograms. In this paper, we adapt their concept of detecting change points in multivariate time series using the sum of thresholded CUSUM statistics. We apply this concept to multivariate locally-stationary processes with Fourier representation with the goal of detecting frequency-specific change points in both autospectra and cross-coherences.

Crucially, our approach recognizes the fact that changes may not be simultaneously synchronized over all EEG channels. Thus, it can distinguish between channelspecific change points and those detectable between channel pairs. Moreover, our method provides insight in terms of conventional frequency-bands and thus gives results that can be easily interpreted by the neuroscientist.

The analysis of brain activity immediately prior to and during epileptic seizure requires a number of characteristics from a statistical method for change-point detection. In light of the complex nature of the observed time series, nonparametric methods have the obvious advantage of avoiding the risk of model misspecification. The method developed in Preuß et al. (2015) is a good example, as it furthermore allows for direct assignment of a change point to channels or brain regions.

However, another important feature for EEG analysis is that the segmentation should be flexible in two respects, (a) change-point candidates should not be restricted to dyadic locations and (b) the method should be applicable to multi-channel data, i.e. we should be able to simultaneously analyze EEG data recorded at different (possibly many) channels, and their interactions. The methods of Cho and Fryzlewicz (2015a), Kirch et al. (2015) and Preuß et al. (2015) are shown to handle data with up to 100, 12 and 5 components, respectively. The data from the epileptic seizure analyzed here is a 21 channel recording from a 10-20 system (which is commonly used in clinical settings) and frequently data from cognitive experiments recorded at 64,128 or 256 channels.

In terms of interpretability, neither Kirch et al. (2015) nor Cho and Fryzlewicz (2015a) are able to identify the specific channels (nor pairs of channels) that drive the change-points - at least not as immediate output - and thus neither method allows 
for interpretation of changes in the dependency between channels. Preuß et al. (2015) emphasize that their method can attribute changes to specific components of the spectral matrix, i.e. autospectra or cross-spectra. However, they illustrate this only for financial time series with length up to 2000, and as this method relies on global optimization it cannot handle long time series such as our data set of over eight minutes (500sec) sampled at $100 \mathrm{~Hz}$ (100 points per second). Analyzing large data is generally a challenge for global segmentation methods, except for dynamic programming approaches. Killick et al. (2012) is in this respect noteworthy, as the algorithm introduced here uses a pruning step to detect multiple change points in univariate data by minimizing a cost function, typically in linear time. However, it is not clear how this method scales to high dimensional data or how it could take the cross-dependence between components of a multivariate time series into account.

To summarize, the proposed method is the first to address the wide range of requirements for the analysis of EEG data that evolves, e.g., before, during and the end of an epileptic seizure. It offers direct interpretability of changes both with respect to spatial location (specific channels) and in terms of specific frequency bands thus producing output that is most useful to neuroscientists and neurologists. It is also computationally fast and scalable, by which we mean that it can be applied to long recordings over many channels. Change points are not restricted to have dyadic locations and are detected in both autospectra and cross-coherences. Simulation studies demonstrate that our method shows a high power and sensitivity even if those occur only in a small subset of time series components for sufficiently long time series.

The application of the proposed method to EEG seizure data illustrates the easy, direct interpretation of the identified changes and provides insights to spectral energy evolution in pre-seizure brain activity. We can identify interesting patterns in the detected changes that point to the existence of pre-seizure biomarkers. Furthermore, we show how the method can support the analysis of epileptic seizure EEG by the neurologist. In the following, we first introduce a model for epileptic seizure EEG as piecewise-stationary process, then propose our frequency-specific change-pint detection method and finally discuss its results in an application to a spontaneous 
seizure event recording. We provide an extensive simulation exercise comparing the proposed method to selected competitors and derive some theoretical results in the supplementary material.

\section{Modeling Epileptic Seizure EEG as Piecewise- Stationary Process}

Epileptic seizure signals can be considered realizations of an underlying brain process containing abrupt changes between different quasi-stationary regimes. As the seizure process unfolds, the energy concentration (autospectrum) undergoes sharp changes at specific frequencies (or frequency bands). Moreover, the strength of the interactions between oscillations at different brain regions, as characterized by cross-coherence, also changes. We formalize this process as follows.

Denote the zero-mean $D$-channel EEG signal to be $\mathbf{X}\left(t^{*}\right)=\left[X_{1}\left(t^{*}\right), \ldots, X_{D}\left(t^{*}\right)\right]^{\prime}$, $t^{*}=\left\{1, \ldots, T^{*}\right\}$. The dimension $D$ is known and, due to physical constraints, must be finite. The set of true change-point locations in rescaled time is $\mathbb{U}=\left\{v_{i} i=\right.$ $1,2, \ldots, N\}$ with, by convention, $v_{0}=0, v_{N+1}=1$. These points define $N+1$ quasistationary segments $\mathcal{I}_{i}^{v}=\left(v_{i-1}, v_{i}\right] \subset(0,1]$. In real time, the change-point locations are defined by $\mathbb{N}=\left\{\eta_{i}, i=1,2, \ldots, N\right\}$ with $\eta_{i}=\left\lceil v_{i} T^{*}\right\rceil$ and, correspondingly $\eta_{0}=0, \eta_{N+1}=T^{*}$ and the $i$-th segment $\mathcal{I}_{i}^{\eta}=\left\{\eta_{i-1}+1, \ldots, \eta_{i}\right\}$ with length $\left|\mathcal{I}_{i}^{\eta}\right|$. The multi-channel EEG signal has the piecewise-stationary representation

$$
\mathbf{X}_{T^{*}}\left(t^{*}\right)=\sum_{i=1}^{N+1} \mathbb{I}_{t^{*}}^{i} \sum_{k=-\left(\left\lfloor\left|\mathcal{I}_{i}^{\eta}\right| / 2\right\rfloor+1\right)}^{\left\lfloor\left|\mathcal{I}_{i}^{\eta}\right| / 2\right\rfloor} \mathbf{A}\left(v_{i}, \omega_{k}\right) \exp \left(j 2 \pi \omega_{k} t^{*}\right) \mathbf{z}^{i}\left(\omega_{k}\right)
$$

where the indicator function $\mathbb{I}_{t^{*}}^{i}=1$ if $t^{*} \in \mathcal{I}_{i}^{\eta}$, and 0 otherwise. Moreover, the vector $\mathbf{z}^{i}\left(\omega_{k}\right)=\left[z_{1}^{i}\left(\omega_{k}\right), \ldots, z_{D}^{i}\left(\omega_{k}\right)\right]^{\prime}$ has zero mean; is independent over frequencies $k$ and blocks $i$ and is identically distributed as standard $D$-variate complex-valued normal with expectation $\mathrm{E} \mathbf{z}^{i}\left(\omega_{k}\right)=\underline{0}$ and covariance $\operatorname{Cov} \mathbf{z}^{i}\left(\omega_{k}\right)=\mathbf{I}(D \times D$ identity matrix $)$. $\mathbf{A}\left(v_{i}, \omega_{k}\right)$ is the transfer function matrix (with dimension $D \times D$ ) defined on rescaled 
time $v_{i} \in(0,1)$ and frequency $\omega_{k}=\frac{2 \pi k}{\left|\mathcal{I}_{i}^{\eta}\right|}$ where $k=-\left\lfloor\left|\mathcal{I}_{i}^{\eta}\right| / 2\right\rfloor+1, \ldots,\left\lfloor\left|\mathcal{I}_{i}^{\eta}\right| / 2\right\rfloor$ satisfying, for each $\omega_{k}, \mathbf{A}\left(u^{*}, \omega_{k}\right)=\mathbf{A}\left(v_{i}, \omega_{k}\right)$ for $u^{*} \in \mathcal{I}_{i}^{v}$, i.e. $\mathbf{A}\left(\cdot, \omega_{k}\right)$ is piecewise constant in both time and frequency.

This process, which contains finite jumps (i.e., jumps of finite size bounded away from zero) at a finite number of change points, belongs to the family of locally stationary processes (Dahlhaus, 2012). Moreover, under Gaussianity, it is fully characterized by the localized spectral matrix, defined on rescaled time $u^{*} \in(0,1)$ with $\left\lceil u^{*} T^{*}\right\rceil=t^{*}$ and frequency $\omega_{k}$,

$$
\mathbf{f}\left(u^{*}, \omega_{k}\right)=\sum_{i=1}^{N+1} \mathbb{I}_{t^{*}}^{i} \mathbf{f}^{i}\left(\omega_{k}\right)
$$

where $\mathbf{f}^{i}\left(\omega_{k}\right)$ is the spectrum of $\mathbf{X}_{T^{*}}\left(t^{*}\right)$ in rescaled time on interval $\mathcal{I}_{i}^{v}$ at frequency $\omega_{k}$. By the one-to-one correspondence of $\eta_{i}$ and $v_{i}, \mathbb{I}_{t^{*}}^{i}=1$ if $u^{*} \in \mathcal{I}_{i}^{v}$ and thus $\mathbf{f}\left(u^{*}, \omega_{k}\right)=\mathbf{f}\left(v, \omega_{k}\right)$ when $u^{*} \in \mathcal{I}_{i}^{v}$. The diagonal elements of $\mathbf{f}\left(u^{*}, \omega_{k}\right)$ represent the univariate localized autospectral densities $f_{d, d}\left(u^{*}, \omega_{k}\right)$ for $d=\{1, \ldots, D\}$ which take the value $f_{d, d}^{i}\left(\omega_{k}\right)$ when $u^{*} \in \mathcal{I}_{i}^{v}$. The off-diagonal entries of $f_{d, d^{\prime}}\left(u^{*}, \omega_{k}\right)$, where $d \neq d^{\prime}$, are localized cross-spectral densities which take the value $f_{d, d^{\prime}}^{i}\left(\omega_{k}\right)$ when $u^{*}$ is in the $i$-th stationary segment in rescaled time.

To facilitate scale-free comparison of the dependence between different pairs of EEG channels across time, we shall study the dependence between channels $d$ and $d^{\prime}$ via the time-varying coherence defined to be

$$
\rho_{d, d^{\prime}}\left(u^{*}, \omega_{k}\right)=\left|f_{d, d^{\prime}}\left(u^{*}, \omega_{k}\right)\right|^{2} /\left(\left|f_{d, d}\left(u^{*}, \omega_{k}\right)\right|\left|f_{d^{\prime}, d^{\prime}}\left(u^{*}, \omega_{k}\right)\right|\right) .
$$

This metric can be interpreted as localized frequency-specific (squared) correlation between a pair of time series components. It measures the strength of linear association between two stationary processes at a particular frequency (or frequency band) and admits values in the range $[0,1]$ indicating weak to strong linear dependence. 
The marginal representation of a single channel (channel $d$ ) is

$$
X_{d}\left(t^{*}\right)=\sum_{i=1}^{N+1} \mathbb{I}_{t^{*}}^{i} \sum_{k=-\left(\left\lfloor\left|\mathcal{I}_{i}^{\eta}\right| / 2\right\rfloor+1\right)}^{\left\lfloor\left|\mathcal{I}_{i}^{\eta}\right| / 2\right\rfloor} a_{d}\left(v_{i}, \omega_{k}\right) \exp \left(j 2 \pi \omega_{k} t^{*}\right) q_{d}^{i}\left(\omega_{k}\right)
$$

where $a_{d}\left(v_{i}, \omega_{k}\right)$ is a scalar complex-value transfer function; and $q_{d}^{i}\left(\omega_{k}\right)$ is iid complexvalued Gaussian with mean 0 and unit variance. The time-varying spectrum for channel $d$ defined on rescaled time $u^{*} \in(0,1)$ with $\left\lceil u^{*} T^{*}\right\rceil=t^{*}$ and frequency $\omega_{k}$,

$$
f_{d}\left(u^{*}, \omega_{k}\right)=\sum_{i=1}^{N+1} \mathbb{I}_{t^{*}}^{i} f_{d}^{i}\left(\omega_{k}\right), \omega \in[-0.5,0.5]
$$

where $f_{d}^{i}\left(\omega_{k}\right)=\left|a_{d}\left(v_{i}, \omega_{k}\right)\right|^{2}$. The derivation is straightforward since $a_{d}\left(v_{i}, \omega_{k}\right) q_{d}^{i}\left(\omega_{k}\right)$ is actually the product of the $d$-th row of the transfer function matrix $\mathbf{A}\left(v_{i}, \omega_{k}\right)$ and the column vector $\mathbf{z}^{i}\left(\omega_{k}\right)$ in Equation (1). The importance of this representation will be made more apparent when we develop our method that examines changes at each frequency band in each channel. This is necessary in order to the evolution of seizure spread from a region (or channel) to another.

Remark (2.1). The process defined in Equation (1) does not require that all channels and all pairs of channels exhibit a change at each of the time points in the set $\mathbb{N}$. Instead, the set $\mathbb{N}$ is a collection of time points at which the autospectrum of at least one channel or the coherence of at least one pair of channels exhibits a change at some frequency. We denote the component-specific subsets $\mathbb{N}^{d, d^{\prime}}=\left\{\eta_{i}^{d, d^{\prime}}, i=\right.$ $\left.1,2, \ldots, N^{d, d^{\prime}}\right\} \subseteq \mathbb{N}$ to be a collection of change points in the autospectrum (when $d=d^{\prime}$ ) or in the cross-coherence (when $d \neq d^{\prime}$ ) where $N^{d, d^{\prime}} \leq N$, and define correspondingly in rescaled time the subsets $\mathbb{U}^{d, d^{\prime}}=\left\{v_{i}^{d, d^{\prime}}, i=1,2, \ldots, N^{d, d^{\prime}}\right\} \subseteq \mathbb{U}$.

We now discuss some assumptions about the underlying process that will help us demonstrate theoretical properties of the frequency-specific change-point detection (FreSpeD) method.

Assumption I The process $\mathbf{X}_{T^{*}}\left(t^{*}\right)$ follows Equation (1) with, for all $d, d^{\prime}: d \neq d^{\prime}$, 


\section{A: Boundedness}

(i.) $f_{d, d}\left(u^{*}, \omega_{k}\right) \leq f^{*}<\infty \quad \forall u^{*}, \omega_{k}$

(ii.) $\rho_{d, d^{\prime}}\left(u^{*}, \omega_{k}\right)<1 \quad \forall u^{*}, \omega_{k}$

\section{B: Minimum separability}

$$
\begin{aligned}
& \text { (i.) } \exists \omega_{k^{*}}:\left|f_{d, d}\left(v_{i}^{d, d}, \omega_{k^{*}}\right)-f_{d, d}\left(v_{i-1}^{d, d}, \omega_{k^{*}}\right)\right| \geq f_{*}>0 \forall i \\
& \text { (ii.) } \exists \omega_{k^{*}}:\left|\rho_{d, d^{\prime}}\left(v_{i}^{d, d^{\prime}}, \omega_{k^{*}}\right)-\rho_{d, d^{\prime}}\left(v_{i-1}^{d, d^{\prime}}, \omega_{k^{*}}\right)\right| \geq \rho_{*}>0 \forall i
\end{aligned}
$$

Remark (2.2). The assumption on boundedness simply restricts the magnitude of both the auto- and cross-spectrum to be bounded above and the minimum separability assumption requires that these changes have to be sufficiently pronounced.

Assumption II For all $v_{i} \in \mathbb{U}$,

$$
\begin{aligned}
& \text { (i.) }\left|v_{i}-v_{i-1}\right| \geq \delta_{T}>0 \\
& \text { (ii.) } \max \left(\frac{v_{i}-v_{i-1}+1}{v_{i+1}-v_{i-1}+1}, \frac{v_{i+1}-v_{i}}{v_{i+1}-v_{i-1}+1}\right) \leq c_{*} \in[1 / 2,1)
\end{aligned}
$$

Remark (2.3). The second set of assumptions restricts change points to be sufficiently distant from another. We allow the minimum change-point distance $\delta_{T}$ to grow with $T^{*}$, but at a lower rate to guarantee that the number of observations in each rescaled interval also grows, as specified in the following section. This assumption is required to ensure identifiability of a change point: if two changes were arbitrarily close, a test would require a sufficient number of observations to detect both change points. The second part is a technical assumption that we can describe as 'balancedness' of the change-point locations. This is necessary to ensure consistency of our CUSUM-type test statistic, which can exhibit erratic behavior close at the edges of the interval on which it is computed.

The process defined in Equation (1) is non-parametric and thus is flexible for representing general time series and robust to misspecification of parametric models 
while still sufficiently powerful in capturing structural changes. Such changes may relate to events such as the presentation of an external stimuli or the build-up to an epileptic seizure. Any change in brain activity will be reflected by a discontinuity in the spectral matrix at the points $\eta_{i}, i \in\{1, \ldots, N\}$, and therefore shows in at least one change in the spectral energy of a component $X_{d, T^{*}}\left(t^{*}\right)$ or a change in the coherence between two components $X_{d, T^{*}}\left(t^{*}\right)$ and $X_{d^{\prime}, T^{*}}\left(t^{*}\right)$.

Remark (2.4). The representation of the time-varying spectral density depends on the frequency $\omega_{k}$. That is, the autospectrum or coherence may remain constant for some frequencies but may change over time for others. Hence change points can be attributed to specific frequencies, for both spectral energy and coherence. This provides additional specific and interpretable results giving a competitive edge over the majority of existing change-point detection methods which compare the full spectral distribution over time, such as Preuß et al. (2015).

In the following section, we develop the FreSpeD method.

\section{Frequency-Specific Change-Point Detection}

The overall aim in this paper is to study the evolution of brain activity in a multichannel epileptic seizure EEG recording $\mathbf{X}_{T^{*}}\left(t^{*}\right)$ with $D$ channels. We characterize the underlying dynamics via change points in time and frequency and for all $D$ autospectra and $D(D-1) / 2$ different pairwise cross-coherences in $\mathbf{X}_{T^{*}}\left(t^{*}\right)$. The first stage in our FreSpeD method is to estimate these unknown autospectral and cross-coherence quantities.

\subsection{Estimation of the spectral quantities}

To obtain information about the spectral properties of $\mathbf{X}_{T^{*}}\left(t^{*}\right)$, it is well-known that some trade-off with the series' temporal resolution is required. Our approach to this is to partition the entire time index $\left\{1, \ldots, T^{*}\right\}$ into short localized intervals (or time blocks) of length $\nu$, which are defined by the boundaries $\mathbb{T}=\left\{t_{l}: t_{l}=l \nu, l=\right.$ 
$1, \ldots, T\}$ where $T=T^{*} / \nu$ (see Figure 1 for an illustration of this partitioning). The equivalent partitioning in rescaled time is $\mathbb{J}=\left\{u_{l}, l=1,2, \ldots, T\right\}$ with $u_{l}=t_{l} / T^{*} \forall l$.

As we describe below, we aggregate information on the short intervals $\left\{t_{l}-\nu+\right.$ $\left.1, \ldots, t_{l}\right\}, t_{l} \in \mathbb{T}$, using standard Fourier estimation principles. The set $\mathbb{T}$ contains all points that we consider as test change-points and therefore ideally the set of true change points $\mathbb{N}$ is contained in $\mathbb{T}$. Assuming that $\mathbb{N} \subset \mathbb{T}$ means that the time series is piecewise stationary with change points located at the points in $\mathbb{T}$, i.e. for any $u^{*}$ s.t. $u^{*} \in\left(u_{l-1}, u_{l}\right], \mathbf{f}\left(u^{*}, \omega_{k}\right)=\mathbf{f}\left(u_{l}, \omega_{k}\right)$ for all $l, k$. This has been assumed in previous works (Adak, 1998; Ombao et al., 2001) and is restrictive but useful when working in practice with large time series such as EEG recordings. However, in our theoretical model, if the change points are located in the interior of the intervals $\left\{t_{l-1}+1, \ldots, t_{l}-1\right\}$ (as opposed to on their boundary points $t_{l}$ ), the effect on the change-point estimation is negligible under Assumption III which will be discussed further in Remark (3.1) below.

Assumption III $T=\mathrm{O}\left(\left(T^{*}\right)^{\Theta}\right)$ with $0.5<\Theta<1$ : as $T^{*} \rightarrow \infty$, then $\nu \rightarrow \infty$ and $T=T^{*} / \nu \rightarrow \infty$, but at a (slightly) lower rate than $T^{*}$. Moreover, the minimum change-point distance $\delta_{T}$ from Assumption II grows proportional to $T / T^{*}$, i.e., $\delta_{T}=$ $\mathrm{O}\left(\left(T^{*}\right)^{\Theta-1}\right)$.

Remark (3.1). Assumption III implies that as $T^{*} \rightarrow \infty$, the partitioning of the rescaled interval (or equivalently the partitioning of the discrete time index $\mathbb{T}$ ) becomes finer but at a lower rate. This ensures that the number of observations, $\nu$, in a localized time block also grows, but slowly, and is natural in spectral analysis since more observations in a time block are needed in order to improve the quality of the estimators of the oscillatory properties of the time series (autospectra and cross-coherence).

Consider the $l$-th time block indexed by $\left\{t_{l-1}+1, \ldots, t_{l}\right\}$. Define the fundamental Fourier frequencies to be $\omega_{k}=k / \nu, k=-\lceil\nu / 2\rceil+1, \ldots,\lceil\nu / 2\rceil$. The $D \times 1$ vector of Fourier coefficients is $\mathrm{d}_{l}\left(\omega_{k}\right)=1 / \sqrt{\nu} \sum_{t^{*}=(l-1) \nu+1}^{l \nu} \mathbf{X}_{T^{*}}\left(t^{*}\right) \exp \left(-j 2 \pi \omega_{k}\left(t^{*}-(l-1) \nu\right)\right)$ 
and the $D \times D$ periodogram matrix is

$$
\mathbf{I}_{l}\left(\omega_{k}\right)=\mathrm{d}_{l}\left(\omega_{k}\right) \mathrm{d}_{l}^{\star}\left(\omega_{k}\right)
$$

where $\mathrm{d}_{l}^{\star}\left(\omega_{k}\right)$ denotes the complex conjugate transpose of $\mathrm{d}_{l}\left(\omega_{k}\right)$.

Remark (3.2). The diagonal entries of the matrix $\mathbf{I}_{l}\left(\omega_{k}\right)$ are the raw periodograms which are the autospectral estimators denoted $\widehat{f}_{d, d}^{R}\left(u_{l}, \omega_{k}\right)=I_{l, d, d}\left(\omega_{k}\right)$. The offdiagonal entries are the raw cross-periodograms which are estimators of the crossspectrum between channels $d$ and $d^{\prime}$, denoted $\widehat{f}_{d, d^{\prime}}^{R}\left(u_{l}, \omega_{k}\right)=I_{l, d, d^{\prime}}\left(\omega_{k}\right)$.

Lemma 1 Let $\widehat{f}_{d, d}^{R}\left(u_{l}, \omega_{k}\right)$ be the autoperiodogram of component $d$ and frequency $\omega_{k}$ computed on time block $l$ that corresponds to the rescaled-time interval $\left(u_{l-1}, u_{l}\right]$. If there is no change point on the interval then $\mathrm{E} \widehat{f}_{d, d}^{R}\left(u_{l}, \omega_{k}\right)=f_{d, d}\left(u_{l}, \omega_{k}\right)$. If the interval contains a change point, denoted $v$, then

$$
\mathrm{E}\left(\widehat{f}_{d, d}^{R}\left(u_{l}, \omega_{k}\right)\right)=\alpha_{1} f_{d, d}\left(u_{l-1}, \omega_{k}\right)+\alpha_{2} f_{d, d}\left(u_{l}, \omega_{k}\right)
$$

where $\alpha_{1}=\left(v-u_{l-1}\right) /\left(u_{l}-u_{l-1}\right)$ and $\alpha_{2}=1-\alpha_{1}$.

Sketch of the proof. In the no change-point situation, the marginal representation of the component at time block $l$ (as described in Equation 2) is $X_{d}\left(t^{*}\right)=$ $\sum_{k} a_{d}\left(v_{l}, \omega_{k}\right) \exp \left(j 2 \pi \omega_{k} t^{*}\right) q_{d}^{l}\left(\omega_{k}\right)$ where $a_{d}\left(v_{l}, \omega_{k}\right)$ is the time-varying transfer function and $q_{d}^{l}\left(\omega_{k}\right)$ is a random variable having a complex-valued standard normal distribution. Recall that the autospectrum of component $d$ on block $l$ and frequency $\omega_{k}$ is $f_{d, d}\left(u_{l}, \omega_{k}\right)=\left|a_{d}\left(v_{l}, \omega_{k}\right)\right|^{2}$ and note that $Q_{d, l, k}=\left|q_{d}^{l}\left(\omega_{k}\right)\right|^{2}$ has a $\chi^{2}(1)$ distribution. Then, the periodogram can be expressed as

$$
\widehat{f}_{d, d}^{R}\left(u_{l}, \omega_{k}\right) \stackrel{D}{=} f_{d, d}\left(u_{l}, \omega_{k}\right) Q_{d, l, k}
$$

where $Q_{d, l, k}$, as noted above, is distributed as $\chi^{2}(1)$. Thus, $\mathrm{E} \widehat{f}_{d, d}^{R}\left(u_{l}, \omega_{k}\right)=f_{d, d}\left(u_{l}, \omega_{k}\right)$. When there is a change point $v$ in $\left(u_{l-1}, u_{l}\right)$ the representation for the time block $l$ is 
a concatenation of two stationary processes with transfer functions $a_{d}\left(v_{l-1}, \omega_{k}\right)$ and $a_{d}\left(v_{l}, \omega_{k}\right)$. It follows that

$$
\widehat{f}_{d, d}^{R}\left(u_{l}, \omega_{k}\right) \stackrel{D}{=} \frac{v-u_{l-1}}{u_{l}-u_{l-1}} f_{d, d}\left(u_{l-1}, \omega_{k}\right) Q_{d, l-1, k}+\frac{u_{l}-v}{u_{l}-u_{l-1}} f_{d, d}\left(u_{l}, \omega_{k}\right) Q_{d, l, k}
$$

where $Q_{d, l-1, k}$ and $Q_{d, l, k}$ are independent $\chi^{2}(1)$ random variables. The result on the expectation follows. As a side remark, the frequency resolutions for blocks of different lengths are also different. For post-processing, in order to match the frequency resolution, we can either upsample the frequencies in the shorter time block or downsample the frequencies in the longer time block. This was adopted in the SLEX methods (see Ombao et al. (2001), Ombao et al. (2005)).

The above lemma indicates that in the presence of a change point which does not coincide with the interval boundary, one can view the interval $\left(u_{l-1}, u_{l}\right]$ as having two piecewise stationary processes. The expectation of the autospectral estimator is a weighted average of the true autospectra of the two processes with weights proportional to the length of time that each of the two processes is present in the interval.

\subsubsection{Practical Considerations}

As noted, our procedure is designed to detect change points in the spectral structure. In practice, we do not search for change points at (singleton) frequencies $\omega_{k}, k=$ $-\lceil\nu / 2\rceil+1, \ldots,\lceil\nu / 2\rceil$. This is motivated by the standard practice in EEG analysis which is based on averages over traditional frequency bands such as the delta, theta, alpha, beta and gamma. In this work, the number of frequency bands $K^{*}$ in a local time block is controlled by the window length, $\nu, K^{*}=\lceil\nu / 2\rceil$. As results based on growing $K^{*}$ are of limited practical use, we fix $\nu$ in the application and simulation studies presented here but provide results for different choices of $\nu$ for the simulation study in the supplementary file.

While the raw periodogram estimator has certain properties which prove useful in the derivation of theoretical results of our change-point estimation method, it is well known that the estimator is inconsistent. We propose the use of the smoothed Welch 
periodogram (Welch, 1967) as spectral estimator in practice. The Welch periodogram for the $l$-th time block indexed by time points $\left\{t_{l-1}+1, \ldots, t_{l}\right\}$ is obtained by breaking this block up into $M$ sub-blocks, computing the periodogram at each sub-block and finally averaging these periodograms. More formally, the smoothed periodogram estimator for the $l$-th time block is

$$
\mathbf{I}_{l}^{S}\left(\omega_{k}\right)=1 / M \sum_{m=1}^{M} \mathrm{~d}_{l, m}\left(\omega_{k}\right) \mathrm{d}_{l, m}^{\star}\left(\omega_{k}\right)
$$

where $\mathrm{d}_{l, m}\left(\omega_{k}\right)=1 / \sqrt{M \nu} \sum_{t^{*}=(l+(m-1) / M-1) \nu+1}^{(l+m / M-1) \nu} \mathbf{X}_{T^{*}}\left(t^{*}\right) \exp \left(-j 2 \pi \omega_{k}\left(t^{*}-(l+(m-\right.\right.$ 1) $(M-1) \nu)$ ) is the Fourier transform for the $m$-th sub-block within the $l$-th block. This differs from the raw periodogram in the additional smoothing through averaging over $M$ equally long sub-blocks on each interval $\left\{t_{l-1}+1, \ldots, t_{l}\right\}$. While this estimator looses frequency resolution in the sense that the number of frequency bands is reduced by the factor $M$, in the numerical study in the supplementary file, it proves to be a more informative input for our change-point detection method than the raw periodogram.

The autospectral estimator for channel $d$ is the $(d, d)$ element of the smoothed periodogram matrix $\widehat{f}_{d, d}^{S}\left(u_{l}, \omega_{k}\right)=I_{l, d, d}^{S}\left(\omega_{k}\right)$. Moreover, using the smoothed periodogram, we can define an estimator for the cross-spectrum between channels $d$ and $d^{\prime}$ as $\widehat{f}_{d, d^{\prime}}^{S}\left(u_{l}, \omega_{k}\right)=I_{l, d, d^{\prime}}^{S}\left(\omega_{k}\right)$. The coherence estimator is then $\widehat{\rho}_{d, d^{\prime}}\left(u_{l}, \omega_{k}\right)=$ $\left|\widehat{f}_{d, d^{\prime}}^{S}\left(u_{l}, \omega_{k}\right)\right|^{2} /\left(\widehat{f}_{d, d}^{S}\left(u_{l}, \omega_{k}\right) \widehat{f}_{d^{\prime}, d^{\prime}}^{S}\left(u_{l}, \omega_{k}\right)\right)$. To be able to apply the same testing concept to the autospectra and cross-coherences, we consider the Fisher-z transforms $\rho_{d, d^{\prime}}^{*}\left(u_{l}, \omega_{k}\right)=1 / 2 \log \frac{1+\rho_{d, d^{\prime}}\left(u_{l}, \omega_{k}\right)}{1-\rho_{d, d^{\prime}}\left(u_{l}, \omega_{k}\right)}$ and $\widehat{\rho}_{d, d^{\prime}}^{*}\left(u_{l}, \omega_{k}\right)=1 / 2 \log \frac{1+\widehat{\rho}_{d, d^{\prime}}\left(u_{l}, \omega_{k}\right)}{1-\widehat{\rho}_{d, d^{\prime}}\left(u_{l}, \omega_{k}\right)}$.

Remark (3.3). In contrast to the raw periodogram, the smoothed periodogram provides us with a set of consistent estimators of the time-varying autospectra and time-varying coherence. Both can be used in testing for change points in real data. While the extension of the theoretical framework to the case of coherence is nontrivial, the focus of this work is to provide a tool that is useful to practitioners to understand brain dynamics. Coherence is crucial in this context and thus is a natural extension of our method. 

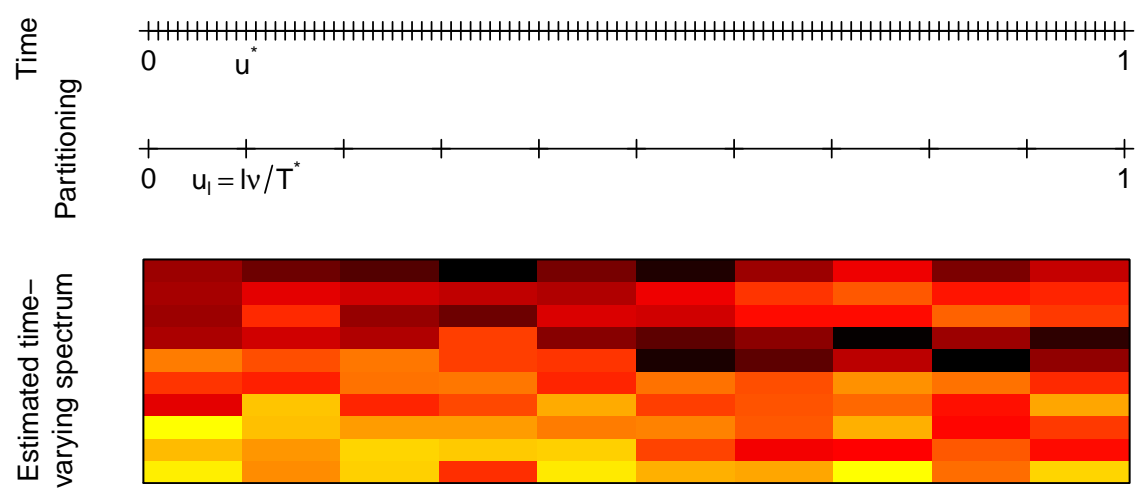

Figure 1: Partitioning of the rescaled-time unit interval into non-overlapping intervals and example of an estimated spectrum (x-axis: time, $\mathrm{y}$-axis: frequency, color indicates level of energy: higher energy corresponds to darker coloring)

\subsection{Estimation of the number and locations of change points}

We present now the heart of the FreSpeD method, the test statistic and its algorithmic framework that allow consistent estimation of change points in time. In many clinical and practical cases, the practitioner will need to know which individual channels and specific pairs of channels exhibit changes (in the autospectra and coherence, respectively), and the frequency bands that are responsible for these changes. Another important requirement in clinical practice is to characterize the spatio-temporal spread of seizure over brain regions. We address these practical challenges by applying the method which we develop to specific channels and pairs of channels. This allows us to track the corresponding sequence of channels that exhibit these changes and thus facilitates the interpretation of spatio-temporal evolution of seizure. As the consequence the minimum change-point distance has to be defined locally (i.e., specific channels and channel pairs) rather than globally.

Assumption II'. For all $v_{i}^{d, d^{\prime}} \in \mathbb{U}^{d, d^{\prime}}$,

$$
\begin{aligned}
& \text { (i.) }\left|v_{i}^{d, d^{\prime}}-v_{i-1}^{d, d^{\prime}}\right| \geq \delta_{T}^{\prime}>0 \\
& \text { (ii.) } \max \left(\frac{v_{i}^{d, d^{\prime}}-v_{i-1}^{d, d^{\prime}}+1}{v_{i+1}^{d, d^{\prime}}-v_{i-1}^{d, d^{\prime}}+1}, \frac{v_{i, d^{\prime}}^{d, d^{\prime}}-v_{i}^{d, d^{\prime}}}{v_{i+1}^{d, d^{\prime}}-v_{i-1}^{d, d^{\prime}}+1}\right) \leq c_{*} \in[1 / 2,1)
\end{aligned}
$$


Moreover, corresponding to Assumption III, we assume that $\delta_{T}^{\prime}$ also grows proportional to $\frac{T}{T^{*}}$ and we will refer to this as Assumption III'. The adaptation to a more flexible spatio-temporal framework is consistent in the simulation and application, but for comparability to our competitor methods that work on global brain patterns we post-process the results as described in the simulation section.

The algorithm is presented for a generic panel of time series $\widehat{z}$ with members $\widehat{z}_{k}=\left\{\widehat{z}_{k}\left(t_{l}\right), t_{l} \in \mathbb{T}\right\}, k \in\left\{1, \ldots, K^{*}\right\}$. These are the block-wise time-varying autospectral and cross-coherence estimates at different frequency bands $k$. The algorithm is applied to estimate change points in the autospectrum of channel $d$ by setting $\widehat{z}_{k}\left(t_{l}\right):=\widehat{f}_{d, d}^{S}\left(u_{l}, \omega_{k}\right) \forall k, l$, and for changes in the coherence between channels $d$ and $d^{\prime}$ to $\widehat{z}_{k}\left(t_{l}\right):=\widehat{\rho}_{d, d}^{*}\left(u_{l}, \omega_{k}\right) \forall k, l$.

We now define the thresholded sum of CUSUM test statistic, computed on the interval $\{(s, e): 1 \leq s<e \leq T\}$ of length $n=e-s+1$, to be

$$
\begin{aligned}
\mathfrak{C}_{s, b, e}(\widehat{z}) & =\sum_{k=1}^{K^{*}} \mathcal{C}_{s, b, e}^{*}\left(\widehat{z}_{k}\right) \mathbb{I}\left(\mathcal{C}_{s, b, e}^{*}\left(\widehat{z}_{k}\right)>\tau_{T}\right) \\
\mathcal{C}_{s, b, e}^{*}\left(\widehat{z}_{k}\right) & =\left|\sqrt{\frac{e-b}{n(b-s+1)}} \sum_{l=s}^{b} \widehat{z}_{k}\left(t_{l}\right)-\sqrt{\frac{b-s+1}{n(e-b)}} \sum_{l=b+1}^{e} \widehat{z}_{k}\left(t_{l}\right)\right| / \widehat{\sigma}_{s, e}\left(\widehat{z}_{k}\right)
\end{aligned}
$$

Here, $\tau_{T}$ is a global threshold and $\mathbb{I}\left(\mathcal{C}_{s, b, e}^{*}\left(\widehat{z}_{k}\right)>\tau_{T}\right)=1$ if $\mathcal{C}_{s, b, e}^{*}\left(\widehat{z}_{k}\right)>\tau_{T}$, zero otherwise. $\widehat{\sigma}_{s, e}\left(\widehat{z}_{k}\right)$ denotes a scaling factor which accounts for the variation of $\widehat{z}_{k}$ on $\{s, \ldots, e\}$. For the autospectra and cross-coherences, respectively,

$$
\begin{aligned}
\widehat{\sigma}_{s, e}\left(\widehat{f}^{S}\left(\omega_{k}\right)\right) & =\frac{1}{n \sqrt{M}} \sum_{l=s}^{e} \widehat{f}^{S}\left(u_{l}, \omega_{k}\right) \\
\widehat{\sigma}_{s, e}\left(\widehat{\rho}^{*}\left(\omega_{k}\right)\right) & =\sqrt{(n-1)^{-1} \sum_{l=s}^{e}\left(\widehat{\rho}^{*}\left(u_{l}, \omega_{k}\right)-n^{-1} \sum_{h=s}^{e} \widehat{\rho}^{*}\left(u_{h}, \omega_{k}\right)\right)^{2}}
\end{aligned}
$$

Remark (3.4). For a single time series $\widehat{z}_{k}$, the statistic $\mathcal{C}_{s, b, e}^{*}\left(\widehat{z}_{k}\right)$ is maximized at the time point $b$ where the contrast between two adjacent blocks $\left[\widehat{z}_{k}(s), \ldots, \widehat{z}_{k}(b)\right]$ and 
$\left[\widehat{z}_{k}(b+1), \ldots, \widehat{z}_{k}(e)\right]$ is maximized. If this contrast, scaled by the process variability, exceeds a threshold $\tau_{T}$, then we consider $b$ a change-point candidate. For any panel of processes $\widehat{z}_{k}, k \in\left\{1, \ldots, K^{*}\right\}$ that can be described as (approximately) piecewiseconstant functions plus noise the statistic $\mathfrak{C}_{s, b, e}(\widehat{z})$ is maximized at one of their change points, and even for large $K^{*}$ this statistic can consistently detect change points that occur only in a small subset of the group while being robust to false positives (Cho and Fryzlewicz, 2015a). Competitor approaches have considered averaging or taking the local pointwise maximum CUSUM statistic (Groen et al., 2013),

$$
\mathfrak{C}_{s, b, e}^{\text {avg }}(\widehat{z})=\frac{1}{K^{*}} \sum_{k=1}^{K^{*}} \mathcal{C}_{s, b, e}^{*}\left(\widehat{z}_{k}\right) \quad \mathfrak{C}_{s, b, e}^{\max }(\widehat{z})=\max _{k=1, \ldots, K^{*}} \mathcal{C}_{s, b, e}^{*}\left(\widehat{z}_{k}\right)
$$

The thresholded-sum CUSUM lies in-between the maximum CUSUM and the average CUSUM (scaled by $K^{*}$ ). The thresholded-sum CUSUM is preferable in settings with large $K^{*}$ in the following sense. Averaging over CUSUMs can yield to what can be labelled false negatives, i.e. undetected sparse change points. At the same time, the maximum CUSUM can lead to false positives, i.e. spurious detection of change points by chance. An interesting property of the thresholded-sum CUSUM of Equation (5) is the assignment of frequency bands to change points. If a point $\hat{\eta}_{i}$ is detected as change point, we can consider the exceedance of the threshold for specific frequency bands $k^{\prime} \in\left\{1, \ldots, K^{*}\right\}$ as evidence that the change occurs at these frequency bands and is not visible in other frequency bands.

Remark (3.5). Within the FreSpeD method, all $D$ autospectra and $D(D-1) / 2$ different coherence pairs of the time series $\mathbf{X}\left(t^{*}\right)$ can be analyzed separately. This is a fast approach to analyzing EEG data with potentially many channels, because the method can be parallelized. For each autospectrum and cross-coherence, we use the thresholded CUSUM statistic within a Binary Segmentation algorithm. In this framework, if we identify a change point on the interval $\{1, \ldots, T\}$ we split the interval at this point, say $b_{0}$, and repeat the procedure on the resulting subintervals $\left\{1, \ldots, b_{0}\right\}$ and $\left\{b_{0}+1, \ldots, T\right\}$.

The algorithm in a pseudo code function is displayed in Figure 3.2. This function 


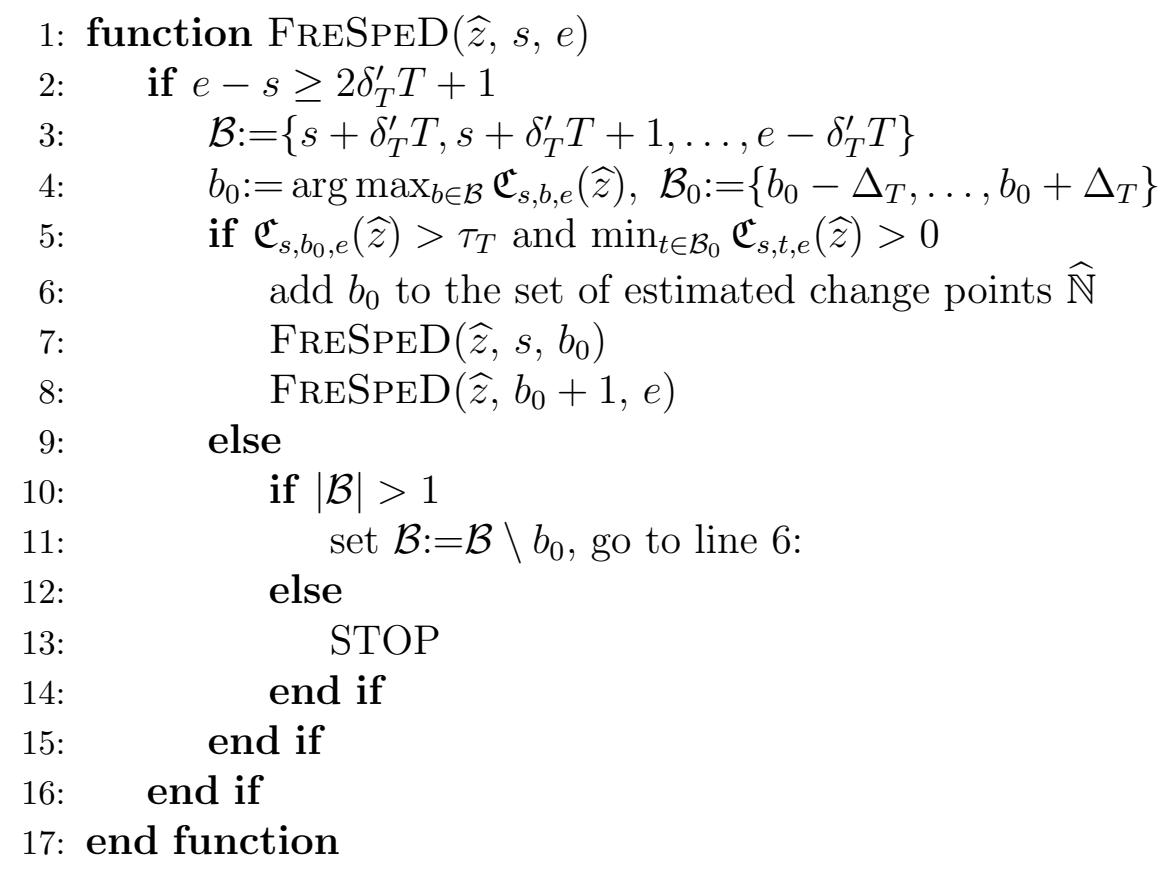

Figure 2: FreSpeD algorithm; initiated by setting $\widehat{z}_{k}\left(t_{l}\right)=\widehat{f}_{d, d}^{S}\left(u_{l}, \omega_{k}\right)$ and $\widehat{z}_{k}\left(t_{l}\right)=$ $\widehat{\rho}_{d, d^{\prime}}^{*}\left(u_{l}, \omega_{k}\right), u_{l} \in \mathbb{J}$ and $s=1, e=T ; \forall d, d^{\prime} \neq d$

is called for $\widehat{z}_{k}\left(t_{l}\right)=\widehat{f}_{d, d}^{S}\left(u_{l}, \omega_{k}\right)$ and $\widehat{z}_{k}\left(t_{l}\right)=\widehat{\rho}_{d, d^{\prime}}^{*}\left(u_{l}, \omega_{k}\right), \forall d, d^{\prime} \neq d$.

The algorithm includes an extra step of evaluating the thresholded CUSUM statistic on the interval $\mathcal{B}_{0}$ of length $\Delta_{T}+1$ around a change-point candidate $b_{0}$ to avoid spurious detections.

We now state theoretical results for consistent change-point detection of the FreSpeD method for autospectral estimates based on the raw periodogram of multichannel EEG data.

Theorem 1 Under Assumptions I, II' and III', as $T^{*} \rightarrow \infty$ there exists $C>0$ and $\kappa>0$ s.t. for $\epsilon_{T}=\log ^{2+\vartheta} T, \tau_{T}=\kappa \log ^{1+\varpi} T$ and $\Delta_{T} \propto \epsilon_{T}$ with $\vartheta>0, \varpi>\vartheta / 2$, the set of change points $\widehat{\mathbb{N}}^{d, d}$ detected by FreSpeD in the autospectrum $\widehat{f}_{d, d}^{R}\left(u_{l}, \omega_{k}\right)$ of channel d satisfies

$$
\operatorname{Pr}\left(\widehat{N}^{d, d}=N^{d, d} ;\left|\widehat{\eta}_{i}^{d, d}-\eta_{i}^{d, d}\right|<C \epsilon_{T} \text { for } i=1, \ldots, N^{d, d}\right) \rightarrow 1 \text { as } T^{*} \rightarrow \infty .
$$


Remark (3.6). The theoretical threshold $\tau_{T}$ and the change-point accuracy bound $\epsilon_{T}$ are derived in the supplementary material using Assumptions I, II' and III'. We note here that the quantities are expressed in terms of $T$ (number of blocks) rather than $T^{*}$ (length of the time series); this simplifies the expressions as the dependency on $\nu$ is not described, but rather follows directly from the equality $T=T^{*} / \nu$.

Remark (3.7). The expression $\left|\hat{\eta}_{i}^{d, d}-\eta_{i}^{d, d}\right|$ is interpreted as the distance between the blocks on which true and estimated change points are located. This distance is bounded above by the quantity $C \epsilon_{T}$ which depends on the total number of blocks. One limitation of the FreSpeD method is the reduced temporal resolution of the change points since it is estimating the block location. This limitation is a consequence of the model used, a block-wise Cramér representation, and appears to be a reasonably small price to pay for ease of interpretability and scalability.

Remark (3.8). As pointed out earlier, the estimated spectral quantities are averaged across frequencies in different frequency bands. These bands in EEG analysis are typically broad. However, there could be practical situations when changes between time blocks could be explained only by changes in the autospectra at a very narrow frequency band. In this setting, it would be helpful to have apriori information from the scientific collaborator. The proposed method is very flexible in terms of fine-tuning the widths of these frequency bands.

Remark (3.9). The concept and theory of thresholded-sum CUSUM statistics for change-point detection was first developed in Cho and Fryzlewicz (2015a) and used in combination with a wavelet-type decomposition of multivariate data. The FreSpeD method was developed specifically for the application to EEG data and differs substantially in two ways. First, FreSpeD uses estimates of local Fourier autospectra and cross-coherences. While there have been developments in the wavelet decomposition of EEG signals, the convention is an interpretation of energy over Fourier frequency bands. Thus, we have tailored our method to be compatible with standard EEG methods and analyses. Second, FreSpeD does not analyze the panel of EEG recordings over channels as a single time series, but rather considers recordings at individual channels and channel-pairs separately. This allows the spatial localization of change points in the sense that we can observe a first change, say, in the seizure 
focal point, followed by changes in neighboring areas. Opposed to this, the algorithm of Cho and Fryzlewicz (2015a) considers the aggregate of Haar wavelet periodograms at all channels and channel pairs simultaneously.

\subsection{Implementation in $R$}

The R package FreSpeD developed by the first author contains an efficient implementation of the FreSpeD method. A first, blinded version of this package is made available as supplementary material that can be included in the review process. Prior to publication on CRAN the package can be installed on Windows from the * ${ }^{*}$ zip-file and requires the installation of dependencies doParallel and foreach, because, in the current $R$ version, dependencies can only be installed automatically if the corresponding files are available in the same location. In addition to the standard code and a set of default parameter values, prior information can be included by adjusting a range of parameters. For example, depending on the available time resolution (sampling rate) and frequency-band resolution of interest, parameter adjustments may be desirable to answer a specific research question focusing, say, on activity at the gamma band $(32-50 \mathrm{~Hz})$. Then coarser resolution can be allowed by decreasing the window length $\nu$. We also point out that the package offers parallelization of the computations over a user-specified number of cores. More details can be found in the package description.

\section{Analysis of Seizure EEG}

We now discuss a number of interesting findings from the application of the FreSpeD method to a seizure recording. This recording captured brain activity of a subject who suffered a spontaneous epileptic seizure while being monitored at the epilepsy center at the University of Michigan. The EEG was sampled at $100 \mathrm{~Hz}(100$ observations per second) and lasted for about $8.3 \mathrm{~min}$ (500sec). The total length is $T=50000$. The EEG was recorded at 21 channels, 19 bipolar scalp electrodes placed according to the 10-20 system and two sphenoidal electrodes placed at the base of 

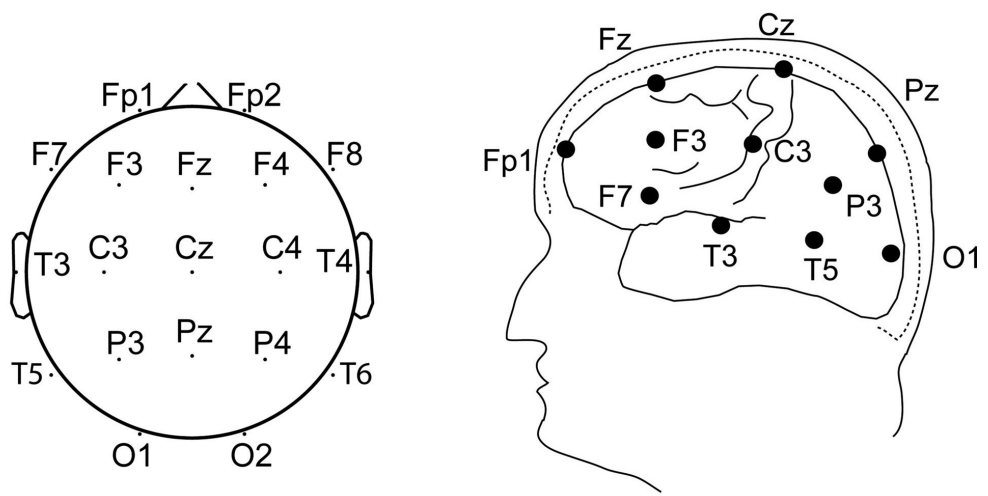

Figure 3: EEG scalp topography of a 10-20 electrode system

the temporal lobe. Figure 3 illustrates the placement of the scalp electrodes. In the 10-20 system, even numbers refer to right and uneven to left hemisphere electrodes. The abbreviations indicate the location on the scalp, from front to back: frontal polar $(\mathrm{Fp})$, frontal $(\mathrm{F})$, central $(\mathrm{C})$, temporal $(\mathrm{T})$, parietal $(\mathrm{P})$ and occipital $(\mathrm{O})$.

The goal of this paper is to capture the dynamic structure of the epileptic seizure process and to identify even the subtle changes in the electrophysiology that precede seizure onset. The FreSpeD method identifies 413 change points over all 21 channels and 210 channel pairs. In total, 105 change points are identified in the channelspecific autospectra and the remaining in pairwise cross-coherences. Figure 4 shows the cumulative sum of detected change points over time, respectively for autospectra, coherences and total. We see that there are barely any changes detected in the preictal period, with the total number of change points rising sharply at around $5.67 \mathrm{~min}$ (roughly at $340 \mathrm{sec}$ or at $t=34000$ ). This sudden increase corresponds with what the attending neurologist identified as seizure onset in this recording. This demonstrates that FreSpeD is able to data-adaptively identify the starting point of an epileptic seizure.

In addition to estimating the time of seizure onset, the estimated change-point distribution can be used to identify the focal point (spatial center) of this particular seizure episode. From the full recording, most change points can be identified in 


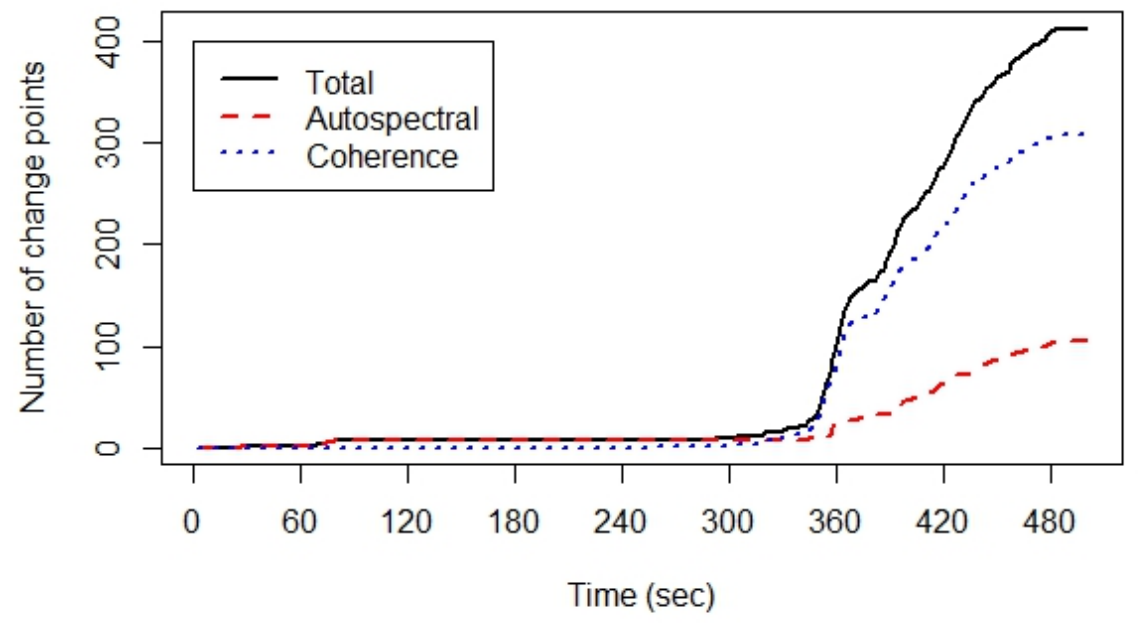

Figure 4: Cumulative sum of detected change points over all channels and/or channel pairs

the autospectra of channels T3 and T5, each of which contains eight change points. Figure 5 illustrates where these changes are located in time and frequency. Again, this is consistent with the neurologist's diagnosis that this patient has left temporal lobe epilepsy and thus electrical activity on this area is projected to the the field on the scalp that covers the T3 and T5 channels.

For a given change point the interpretation of thresholded CUSUM statistics is not straightforward. The number of frequency bands where a change is detectable is a more robust and easily interpretable measure which illustrates the intensity or spread of a change. Both channels, T3 and T5, have more and less spread change points around the seizure onset. However, overall, there are more frequency bands showing a change at channel T3 - the primary focal point according to the neurologist. We note that this data-adaptive seizure localization by the FreSpeD method can be of great value to neuroscientists: an automated mechanism to support and validate a neurologist's judgement diminishes the risk of human error in the visual inspection of EEG traces and is more time efficient. 


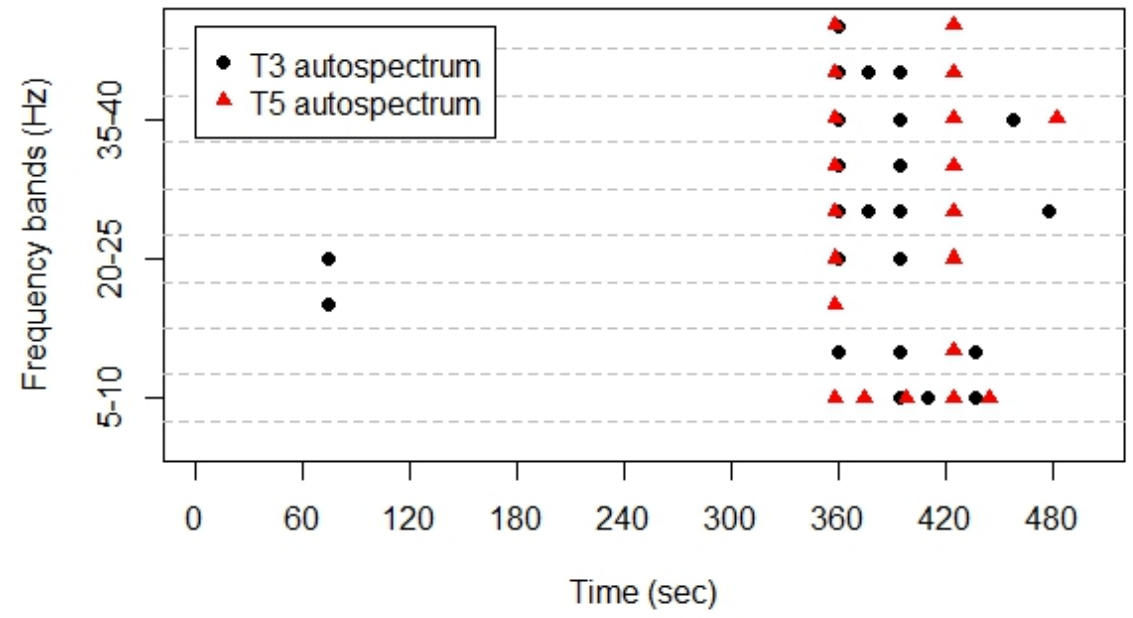

Figure 5: Change points in the autospectra of channels T3 and T5 in time (x-axis) vs frequency (y-axis)

We now examine the occurrence of change points across frequency bands shown in Table 4. Most often the method was able to identify changes in the autospectra and coherence at low frequency ranges, most pronounced in the theta band. While we identify comparably few changes at higher frequencies, the cumulative absolute magnitude is larger and thus more emphatic. This is in-line with visual inspection and literature on seizure data analysis (Worrell et al., 2004; Jiruska et al., 2010). During normal brain states, energy concentration at high frequencies is low, but we observe a pronounced sudden increase at seizure onset. However, the observation of greater number but smaller magnitude (and therefore possibly not visually detectable) of change points at lower frequencies has not received much attention to date. This suggests that low-frequency energy and coherence vary more frequently immediately before and during epileptic seizure.

The evolution of brain processes leading up to a seizure onset is of high interest in the context of seizure warning systems. With the general aim of an improved understanding of changes in the spectral features of the brain process we now focus 


\begin{tabular}{|c|c|c|c|c|c|c|}
\hline $\begin{array}{c}\text { Frequency } \\
\text { band }\end{array}$ & $\begin{array}{l}\text { Start } \\
\text { freq. }\end{array}$ & $\begin{array}{l}\text { End } \\
\text { freq. }\end{array}$ & \multicolumn{2}{|c|}{$\begin{array}{l}\text { Prop. of total change points } \\
\text { autospectra } \mid \text { coherences }\end{array}$} & \multicolumn{2}{|c|}{ Prop. of change magnitude } \\
\hline theta & 5 & 10 & 58.1 & 32.2 & 54.8 & 51.3 \\
\hline alpha & 10 & 15 & 33.3 & 24.0 & 23.1 & 32.4 \\
\hline beta & 15 & 20 & 24.8 & 23.5 & 15.0 & 30.6 \\
\hline beta & 20 & 25 & 24.8 & 18.6 & 28.3 & 28.6 \\
\hline beta & 25 & 30 & 28.6 & 16.7 & 41.2 & 31.2 \\
\hline gamma & 30 & 35 & 30.5 & 18.4 & 50.5 & 36.9 \\
\hline gamma & 35 & 40 & 38.1 & 23.2 & 73.5 & 50.3 \\
\hline gamma & 40 & 45 & 41.0 & 24.0 & 80.6 & 55.6 \\
\hline gamma & 45 & 50 & 35.2 & 23.7 & 83.9 & 55.3 \\
\hline
\end{tabular}

Table 1: Frequency-specific proportion of change points and change magnitude. Change magnitude is measured as sum over thresholded CUSUM statistics, over time, frequency and components or component pairs

on highlighting a number of findings regarding preictal EEG. However, it should be noted that seizure precursors can vary between different seizures of one patient and between patients. For this particular data, the FreSpeD method was able to detect very subtle changes that are not visually obvious to the neurologist. Firstly, a cluster of pre-ictal change points is identified at the very start of the recording (Figure 6 ). This cluster consists of seven channel-specific changes in the autospectrum and contains no coherence change. Six of the detected changes occur between 1.00min and $1.30 \mathrm{~min}(60-78 \mathrm{sec})$ from the start of the recording.

There is also a very first change within the first 30sec of recording. In light of the instability of the CUSUM statistic at the very borders of the interval and the small magnitude of the detected change, it can be debated if this first change point at 30sec is spurious. However, just as each of the following six change points it is detected in the lower end of the beta-band. Within this cluster of six autospectra changing shortly after another, all channels are located in the back half of the brain and none in the frontal lobe. The largest change in terms of magnitude is detected in channel T5, whereas channel T3 is the only channel with a change detected also in the mid-range of the beta-band, i.e. here the change is more spread over frequencies. While the temporal lag to the seizure onset makes it difficult to argue in favor of a 
direct link, the fact that FreSpeD independently picks up changes at six channels, all early in the second minute of the recording, reflects event-related global brain activity changes.

A second cluster can be identified at approximately $1.30 \mathrm{~min}$ (78sec) immediately prior to seizure onset. Here, the FreSpeD method detects 13 changes in the coherence of several channel pairs and, quite interestingly and different to the fist observed cluster, none in channel-specific autospectra. As illustrated in Figure 6, FreSpeD captures these changes in coherence that can be described as slowly building up, culminating into the full-blown seizure onset. Furthermore, the changes are not confined at a local spatial region. Rather these were evenly distributed over the brain with 15 channels being involved, 2 centrally located, 7 on the left and 6 on the right hemisphere. The changes are mostly visible in the theta/low alpha and gamma frequency bands. At the time of writing, such an observation has not received attention by researchers. In fact, these changes were not detected in other works that previously analyzed this same data set such as Ombao et al. $(2002,2005)$ and Davis et al. (2006). The FreSpeD method demonstrates that, even prior to seizure onset, dependencies between brain regions already change, both within the seizure area and with more distant regions. As already noted, these changes are not visually identifiable. In fact, many epileptologists determine seizure onset by visual inspection of EEG traces and hence can only identify changes at single channel variance and spectral energy. The observation made here could potentially change the paradigm for seizure characterization that it is beyond abnormal local changes. It is worthwhile to point out that this observation is an immediate output of out method, as the detected changes have a direct interpretation with respect to frequency band and topological location.

\section{Summary and Future Directions}

This paper demonstrates that the FreSpeD method effectively detects change points in multi-channel EEG traces and hereby can help improve our understanding of complex brain activity such as epileptic seizure. In contrast to other methods, changes 


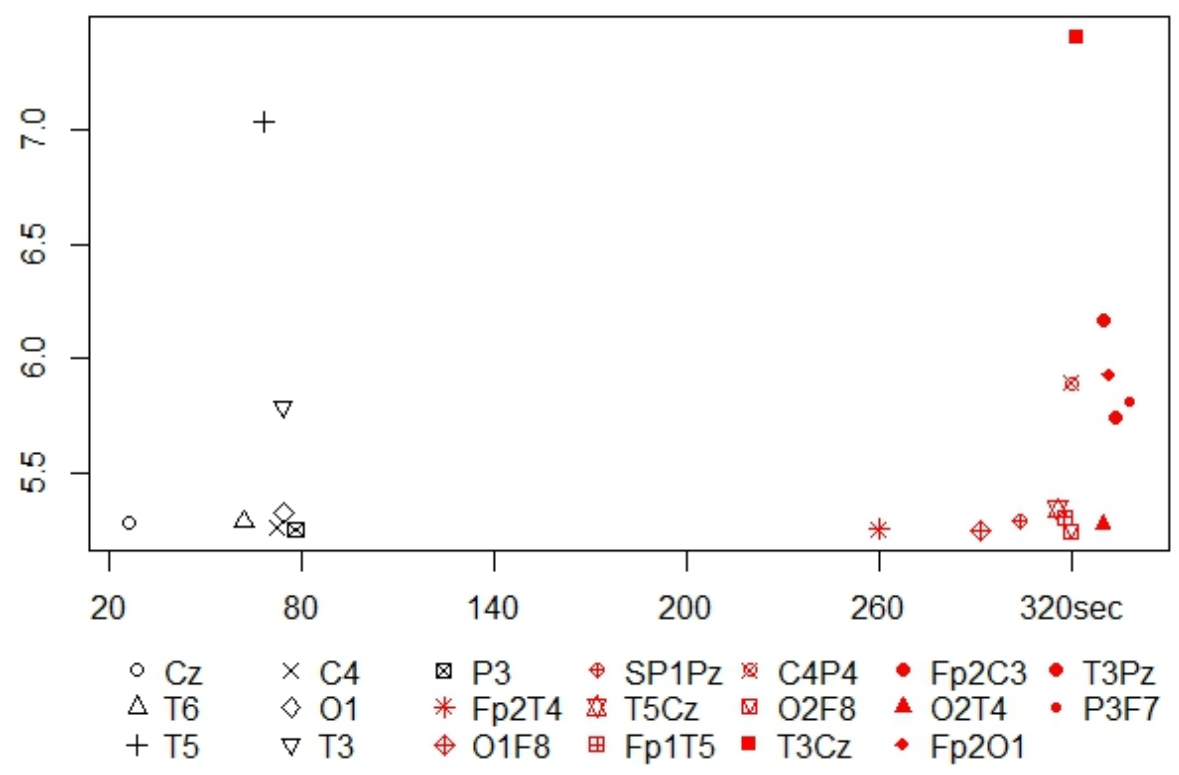

Figure 6: Preictal changes at all autospectra and cross-coherences. The x-axis displays time (with the seizure onset at around 340sec), the y-axis shows the level of the test statistics at the change points, averaged by number of frequencies in which a change is detected. 
detected by FreSpeD can be directly attributed to specific frequency bands; autospectra of specific EEG channels; and cross-coherences of specific pairs of channels. Moreover, since the temporal sequence of change points for each individual channels are estimated, our method indirectly gives a picture of how seizure evolves and propagates from one localized region (or channel) to others in the brain network. To the best of our knowledge, this direct and detailed interpretability is unique to the FreSpeD method and thus makes it a particularly attractive tool to brain scientists and neurologists.

We applied the FreSpeD method to the multi-channel EEG recording of a spontaneous epileptic seiszure and changes in cross-coherence immediately before seizure onset were identified. We emphasize that these changes are subtle and were, in fact, not detected by the methods that previously analyzed the same EEG data. These changes are not sufficiently obvious to be detected through mere eyeballing. Thus the FreSpeD method gives additional insights into the complex nature of an epileptic seizure and is a new approach that can potentially identify seizure precursors. Furthermore, the FreSpeD method estimates the timing of seizure onset and the spatial focal point consistently with the neurologist's diagnosis. This data-adaptive seizure analysis via FreSpeD can be used to check the physicians' diagnoses and thus can reduce the risk of human error.

The comparison of FreSpeD against a parametric and a nonparametric changepoint detection method for multi-channel EEG data in a simulation study in the supplementary material shows the robustness of our approach to model misspecification. It also underlines that the FreSpeD method works well even when the dimensionality increases. In addition, due to possible parallelization of the method and a computationally attractive algorithmic structure, FreSpeD is fast even when the number of observations in time and the number of channels is large.

Based on the approach presented here, there are a number future research directions that can be pursued. First, multi-channel EEG data with a large number of channels tend to show a high level of collinearity. This calls for transforming the data into a lower dimensional set of signals via some appropriate transformation that effectively captures the change-points dynamics of the original data. One could then 
apply the FreSpeD method to the summarized lower dimensional signal to discover the change-points in the original data. This would be in analogous to the recent work of Aston et al. (2012) but the emphasis of our work would be on detecting changes on the spectral, rather than time domain, quantities.

In the current implementation, FreSpeD is designed to detect change points in the autospectra and in (classical) coherence. Coherence at the same frequency may not completely characterize the dependence in complex data such as multi-channel EEG. A more general measure of dependence is evolutionary dual-frequency coherence where one can examine dependence between, say, the alpha oscillation in one channel and the theta oscillation in another (Gorrostieta et al., 2012). Thus, building on FreSpeD one can develop a procedure for detecting change points in dualfrequency coherence to understand the evolutionary dependence structure between EEG channels or brain regions.

\section{References}

S Adak. Time-dependent spectral analysis of nonstationary time series. Journal of the American Statistical Association, 93(444):1488-1501, 1998.

G Alarcon, CD Binnie, RDC Elwes, and CE Polkey. Power spectrum and intracranial EEG patterns at seizure onset in partial epilepsy. Electroencephalography and Clinical Neurophysiology, 94(5):326-337, 1995.

John AD Aston, Claudia Kirch, et al. Evaluating stationarity via change-point alternatives with applications to fmri data. The Annals of Applied Statistics, 6 (4):1906-1948, 2012.

NA Blondin and DM Greer. Neurologic prognosis in cardiac arrest patients treated with therapeutic hypothermia. The Neurologist, 17(5):241-248, 2011.

D Bosq. Nonparametric statistics for stochastic processes: estimation and prediction, volume 110. Springer Science \& Business Media, 2012. 
J Chen and K Gupta. Parametric Statistical Change Point Analysis: With Applications to Genetics, Medicine, and Finance. Boston: Birkhäuser Boston, 2012.

Y Chen, WK Härdle, and U Pigorsch. Localized realized volatility modeling. Journal of the American Statistical Association, 105(492):1376-1393, 2010.

H Cho and P Fryzlewicz. Multiple-change-point detection for high dimensional time series via sparsified binary segmentation. Journal of the Royal Statistical Society: Series B (Statistical Methodology), 77(2):475-507, 2015a.

H Cho and P Fryzlewicz. Corrections on multiple-change-point detection for high dimensional time series via sparsified binary segmentation. 2015b.

I Cribben, R Haraldsdottir, LY Atlas, TD Wager, and MA Lindquist. Dynamic connectivity regression: determining state-related changes in brain connectivity. Neuroimage, 61(4):907-920, 2012.

I Cribben, TD Wager, and MA Lindquist. Detecting functional connectivity change points for single-subject fMRI data. Frontiers in Computational Neuroscience, 7, 2013.

R Dahlhaus. Locally stationary processes. Handbook of Statistics, Time Series Analysis: Methods and Applications, pages 351-408, 2012.

RA Davis, TCM Lee, and GA Rodriguez-Yam. Structural break estimation for nonstationary time series models. Journal of the American Statistical Association, 101:223-239, 2006.

C Gorrostieta, H Ombao, R Prado, S Patel, and E Eskandar. Exploring dependence between brain signals in a monkey during learning. Journal of Time Series Analysis, 33(5):771-778, 2012.

JJJ Groen, G Kapetanios, and S Price. Multivariate methods for monitoring structural change. Journal of Applied Econometrics, 28(2):250-274, 2013. 
P Jiruska, J Csicsvari, AD Powell, JE Fox, W-C Chang, M Vreugdenhil, X Li, M Palus, AF Bujan, RW Dearden, and JGR Jefferys. High-frequency network activity, global increase in neuronal activity, and synchrony expansion precede epileptic seizures in vitro. The Journal of Neuroscience, 30(16):5690-5701, 2010.

NL Johnson and S Kotz. Distributions in Statistics: Continuous Univariate Distributions: Vol.: 1. Houghton Mifflin, 1970.

A Kaplan, J Röschke, B Darkhovsky, and J Fell. Macrostructural eeg characterization based on nonparametric change point segmentation: application to sleep analysis. Journal of Neuroscience Methods, 106(1):81-90, 2001.

R Killick, P Fearnhead, and IA Eckley. Optimal detection of changepoints with a linear computational cost. Journal of the American Statistical Association, 107 (500):1590-1598, 2012.

C Kirch, B Muhsal, and H Ombao. Detection of changes in multivariate time series with application to EEG data. Journal of the American Statistical Association, (just-accepted):-, 2015.

TS Kumar, V Kanhangad, and RB Pachori. Classification of seizure and seizure-free EEG signals using multi-level local patterns. In Digital Signal Processing, 2014 19th International Conference on, pages 646-650. IEEE, 2014.

$\mathrm{H}$ Ombao and S van Bellegem. Evolutionary coherence of nonstationary signals. Signal Processing, IEEE Transactions on, 56(6):2259-2266, 2008.

H Ombao, J Raz, R von Sachs, and W Guo. The SLEX model of a non-stationary random process. Annals of the Institute of Statistical Mathematics, 54:171-200, 2002.

H Ombao, R von Sachs, and W Guo. SLEX analysis of multivariate nonstationary time series. Journal of the American Statistical Association, 100(470):519-531, 2005. 
Hernando C Ombao, Jonathan A Raz, Rainer von Sachs, and Beth A Malow. Automatic statistical analysis of bivariate nonstationary time series. Journal of the American Statistical Association, 96(454):543-560, 2001.

P Preuß, R Puchstein, and H Dette. Detection of multiple structural breaks in multivariate time series. Journal of the American Statistical Association, 110 (510):654-668, 2015.

ME Saab and J Gotman. A system to detect the onset of epileptic seizures in scalp EEG. Clinical Neurophysiology, 116(2):427-442, 2005.

SE Schmitt, K Pargeon, ES Frechette, LJ Hirsch, J Dalmau, and D Friedman. Extreme delta brush: A unique EEG pattern in adults with anti-NMDA receptor encephalitis. Neurology, 79(11):1094-1100, 2012.

FT Sun, LM Miller, and M D'Esposito. Measuring interregional functional connectivity using coherence and partial coherence analyses of fMRI data. Neuroimage, 21(2):647-658, 2004.

J Terrien, G Germain, C Marque, and B Karlsson. Bivariate piecewise stationary segmentation; improved pre-treatment for synchronization measures used on nonstationary biological signals. Medical Engineering \& Physics, 35(8):1188-1196, 2013.

Trans-Cranial-Technologies. 10/20 system positioning. 2012. URL https:// www.trans-cranial.com/local/manuals/10_20_pos_man_v1_0_pdf.pdf. last checked on 05/09/2017.

ES Venkatraman. Consistency results in multiple change-point problems. PhD thesis, Department of Statistics, Stanford University, 1992.

$\mathrm{P}$ Welch. The use of fast Fourier transform for the estimation of power spectra: a method based on time averaging over short, modified periodograms. IEEE Transactions on audio and electroacoustics, pages 70-73, 1967. 
GA Worrell, L Parish, SD Cranstoun, R Jonas, G Baltuch, and B Litt. Highfrequency oscillations and seizure generation in neocortical epilepsy. Brain, 127 (7):1496-1506, 2004.

\section{A Proof of Theorem 1}

The structure of the proof is as follows. We first show the consistency of change-point detection for estimated spectral energy at a single frequency band $\omega_{k}$. Then we extend this to the thresholded sum statistic of Equation (5). As FreSpeD runs on each channel (and channel pair) separately, we drop the channel-indicating subscript $d$ in the following. The results are derived for the true autospectra $z_{k}\left(t_{l}\right):=f_{d, d}\left(u_{l}, \omega_{k}\right)$ and the autospectral estimate computed from the raw periodogram, $\widehat{z}_{k}\left(t_{l}\right):=\widehat{f}_{d, d}^{R}\left(u_{l}, \omega_{k}\right)$ for all frequencies $k$ and time points $t_{l} \in \mathbb{T}$, as input for FreSpeD.

\section{A.1 CUSUM for a single frequency band $\omega_{k}$}

Let us first introduce some notation. On an interval $\{s, \ldots, e\}, 1 \leq s<e \leq T$ we define the statistics

$$
\mathcal{C}_{s, b, e}\left(\widehat{z}_{k}\right)=\left|\sqrt{\frac{e-b}{n(b-s+1)}} \sum_{l=s}^{b} \widehat{z}_{k}\left(t_{l}\right)-\sqrt{\frac{b-s+1}{n(e-b)}} \sum_{l=b+1}^{e} \widehat{z}_{k}\left(t_{l}\right)\right|
$$

for the time-varying estimator of the spectral quantity and, correspondingly, $\mathcal{C}_{s, b, e}\left(z_{k}\right)$ for the true quantities, with $n=e-s+1$. Note that this is the CUSUM statistic of Equation (6) up to scaling: $\mathcal{C}_{s, b, e}(x)=\sigma_{s, e}(x) \mathcal{C}_{s, b, e}^{*}(x)$.

At any stage of the algorithm, for $1 \leq i_{1}<i_{2} \leq N, \eta_{i_{1}} \leq s<\eta_{i_{1}+1}<\cdots<\eta_{i_{2}}<e \leq$ $\eta_{i_{2}+1}$. If at any stage there are change points on $\{s, \ldots, e\}$ then both of the following conditions hold. This will become obvious on the next pages.

$$
\begin{array}{r}
s<\eta_{i_{1}+i}-c_{1} \delta_{T}^{\prime} T<\eta_{i_{1}+i}+c_{1} \delta_{T}^{\prime} T<e \text { for some } 1 \leq i \leq i_{2}-i_{1} \\
\quad \max \left\{\min \left(\eta_{i_{1}+1}-s, s-\eta_{i_{1}}\right), \min \left(\eta_{i_{2}+1}-e, e-\eta_{i_{2}}\right)\right\} \leq c_{2} \epsilon_{T}
\end{array}
$$


Here, $c_{1}, c_{2}$ are positive constants and $\epsilon_{T}$ is as defined in Theorem 1. The following Lemmata are adapted from Cho and Fryzlewicz (2015a) and the corrections available in Cho and Fryzlewicz (2015b).

Lemma 2 Let $\{s, \ldots$, e $\}$ satisfy Equation (8). Then there exists a $i^{*}$ where $1 \leq i^{*} \leq$ $i_{2}-i_{1}$ such that for a positive constant $C$,

$$
\left|\mathcal{C}_{s, \eta_{i_{1}+i^{*}, e}}\left(z_{k}\right)\right| \geq C \delta_{T}^{\prime} \sqrt{T}
$$

Proof For the single change-point case, $i^{*}=1, z_{k}\left(t_{l}\right)$ is piecewise-constant before and after $\eta_{i_{1}+i^{*}}$ on $\{s, \ldots, e\}$. Thus

$$
\begin{aligned}
\left|\mathcal{C}_{s, \eta_{i_{1}+i^{*}}, e}\left(z_{k}\right)\right| & =\sqrt{\left(\eta_{i_{1}+i^{*}}-s+1\right)\left(e-\eta_{i_{1}+i^{*}}\right)} / \sqrt{n}\left|z_{k}\left(\eta_{i_{1}+i^{*}}+1\right)-z_{k}\left(\eta_{i_{1}+i^{*}}\right)\right| \\
& \geq z_{*} c_{1} \delta_{T}^{\prime} \sqrt{T}
\end{aligned}
$$

where $z_{*}$ is the lower bound imposed on change sizes in the corresponding spectral quantities and the inequality follows from Assumptions I and II'. For the case of multiple change points, by Assumption I for any interval satisfying the condition of Equation (8) there exists at least one $i^{*}$ s.t.

$$
\frac{1}{\eta_{i_{1}+i^{*}}-s+1} \sum_{l=s}^{\eta_{i_{1}+i^{*}}} z_{k}\left(t_{l}\right)-\frac{1}{e-\eta_{i_{1}+i^{*}}} \sum_{l=\eta_{i_{1}+i^{*}+1}}^{e} z_{k}\left(t_{l}\right)
$$

is bounded away from zero. This generates the same situation as for the single change-point case and thus Lemma 2 is shown.

Lemma 3 Under the condition of Equation (8), there exists a $C>0$ such that for points $b \in\{s, \ldots, e\}$ with $\left|\eta_{i_{1}+i}-b\right|>c_{0} \epsilon_{T}$ for some $k$ and $\mathcal{C}_{s, \eta_{i_{1}+i}, e}\left(z_{k}\right)>\mathcal{C}_{s, b, e}\left(z_{k}\right)$,

$$
\mathcal{C}_{s, \eta_{i_{1}+i}, e}\left(z_{k}\right)>\mathcal{C}_{s, b, e}\left(z_{k}\right)+C \epsilon_{T} \delta_{T}^{\prime} / T \mathcal{C}_{s, \eta_{i_{1}+i}, e}\left(z_{k}\right)
$$

Proof Without loss of generality, let $\eta \equiv \eta_{i_{1}+i}<b$. The proof follows directly from the proof of Lemma 2.6, Case 2 in Venkatraman (1992), which is at three full pages 
rather lengthy. Essentially, one can show that as $T \rightarrow \infty$, the difference $\mathcal{C}_{s, \eta_{i_{1}+i}, e}\left(z_{k}\right)-$ $\mathcal{C}_{s, b, e}\left(z_{k}\right)$ is bounded below by a term is dominated by what Venkatraman (1992) calls $E_{1 l}$. In our notation and noting that $\delta_{T}^{\prime} T>2 c_{0} \epsilon_{T}$ for large $T$,

$$
\begin{aligned}
& E_{1 l} \\
& =\frac{\left(\delta_{T}^{\prime} T-c_{0} \epsilon_{T}\right) c_{0} \epsilon_{T} \sqrt{n_{\eta}+n_{e}+\delta_{T}}\left[\left(\eta_{i_{1}+i}-s+1\right)^{-1} \sum_{l=s}^{\eta_{i_{1}+i}} z_{k}\left(t_{l}\right)-\left(e-\eta_{i_{1}+i}\right)^{-1} \sum_{l=\eta_{i_{1}+i}+1}^{e} z_{k}\left(t_{l}\right)\right]}{\sqrt{n_{\eta}\left(n_{e}+\delta_{T}^{\prime} T\right)\left(n_{\eta}+c_{0} \epsilon_{T}\right)\left(n_{e}+\delta_{T}-c_{0} \epsilon_{T}\right)}\left(\sqrt{\left(n_{\eta}+c_{0} \epsilon_{T}\right)\left(n_{e}+\delta_{T}^{\prime} T-c_{0} \epsilon_{T}\right)}+\sqrt{n_{\eta}\left(n_{e}+c_{0} \epsilon_{T}\right)}\right)} \\
& \geq C \epsilon_{T} \delta_{T}^{\prime} / T \mathcal{C}_{s, \eta_{i_{1}+i}, e}\left(z_{k}\right)
\end{aligned}
$$

where $n_{\eta}=\eta_{i_{1}+i}-s+1$ and $n_{e}=e-\eta_{i_{1}+i}-\delta_{T}^{\prime} T$.

Lemma 4 Let $\mathcal{D}=\left\{(s, b, e): 1 \leq s<e \leq T ; n=e-s+1 \geq \delta_{T}^{\prime} T ; \max (b-s+\right.$ $\left.1, e-b) \leq n c_{*}\right\}$, where $c_{*}$ as in Assumption II'. Then as $T \rightarrow \infty$,

$$
\operatorname{Pr}\left(\max _{(s, b, e) \in \mathcal{D}}\left|\mathcal{C}_{s, b, e}\left(\widehat{z}_{k}\right)-\mathcal{C}_{s, b, e}\left(z_{k}\right)\right|>\log T\right) \rightarrow 0
$$

Proof For the autospectral estimator from the raw periodogram we have $\widehat{f}_{d, d}^{R}\left(u_{l}, \omega_{k}\right)=$ $f_{d, d}\left(u_{l}, \omega_{k}\right) Q_{l, k}$ where $Q_{l, k}$ is $\chi^{2}(1)$, if the interval $\left(u_{l-1}, u_{l}\right]$ contains no change point. If the interval contains some change point $v, \widehat{f}_{d, d}^{R}\left(u_{l}, \omega_{k}\right)=\alpha_{1} f_{d, d}\left(u_{l-1}, \omega_{k}\right) Q_{l-1, k}+$ $\alpha_{1} f_{d, d}\left(u_{l}, \omega_{k}\right) Q_{l, k}$ with $\alpha_{1}=\left(v-u_{l-1}\right) /\left(u_{l}-u_{l-1}\right)$ and $\alpha_{2}=1-\alpha_{1}$ and where $Q_{l-1, k}$ and $Q_{l, k}$ independent.

Using the simplified notation of this proof, consider

$$
\begin{aligned}
& \operatorname{Pr}\left(\left|\sum_{l=s}^{e} c\left(t_{l}\right) z_{k}\left(t_{l}\right)\left(Q_{l, k}-1\right)\right|>\sqrt{n} \log T\right) \\
& =\operatorname{Pr}\left(\left|\sum_{l=s}^{e} c\left(t_{l}\right) z_{k}\left(t_{l}\right)\left(U^{2}\left(t_{l}\right)-1\right)\right|>\sqrt{n} \log T\right)
\end{aligned}
$$

with $c\left(t_{l}\right)=\sqrt{e-b} / \sqrt{b-s+1}$ if $s \leq l \leq b$ and $c\left(t_{l}\right)=-\sqrt{b-s+1} / \sqrt{e-b}$ if $b<$ $l \leq e$, which are finite on $\mathcal{D}$ as $\left|c\left(t_{l}\right)\right| \leq c^{*} \equiv \sqrt{c_{*} /\left(1-c_{*}\right)}<\infty . Q_{l, k}-1=U^{2}\left(t_{l}\right)-1$ where $U\left(t_{l}\right)$ are independent standard normal random variables. By standard results 
there exists $C>0$ s.t. $\mathrm{E}\left(\left|U^{2}\left(t_{l}\right)-1\right|\right)^{j} \leq C^{j-2} j$ ! $\mathrm{E}\left(\left|U^{2}\left(t_{l}\right)-1\right|\right)^{2}$ (see e.g. Johnson and Kotz, 1970). Thus by Bernstein's inequality (Bosq, 2012),

$$
\begin{aligned}
& \operatorname{Pr}\left(\left|\sum_{l=s}^{e} c\left(t_{l}\right) z_{k}\left(t_{l}\right)\left(U^{2}\left(t_{l}\right)-1\right)\right|>\sqrt{n} \log T\right) \\
& \leq 2 \exp \left\{-\frac{1 / 2 n \log ^{2} T}{2 \sum_{l=s}^{e}\left(c\left(t_{l}\right) z_{k}\left(t_{l}\right)\right)^{2}+C \max _{l} c\left(t_{l}\right) z_{k}\left(t_{l}\right) \sqrt{n} \log T}\right\}
\end{aligned}
$$

As $\left|c\left(t_{l}\right)\right| \leq c^{*}$ and $\left|z_{k}\left(t_{l}\right)\right| \leq z^{*}$, it follows that

$$
\begin{aligned}
& \operatorname{Pr}\left(\max _{(s, b, e) \in \mathcal{D}}\left|\mathcal{C}_{s, b, e}\left(\widehat{z}_{k}\right)-\mathcal{C}_{s, b, e}\left(z_{k}\right)\right|>\sqrt{n} \log T\right) \\
& \leq 2 \sum_{(s, b, e) \in \mathcal{D}} \exp \left\{-\frac{1 / 2 n \log ^{2} T}{2 n\left(c^{*} z^{*}\right)^{2}+C c^{*} z^{*} \sqrt{n} \log T}\right\} \\
& \leq 2 T^{3} \exp \left(-C \log ^{2} T\right)
\end{aligned}
$$

The last converges to zero as we assume $n \geq \delta_{T}^{\prime} T \geq \log T$ and that all constants are finite.

Lemma 5 Under Equations (8) and (9), for $\mathcal{D}_{s, e}=\{s<l<e ; n=e-s+1 \geq$ $\left.\delta_{T}^{\prime} T ; \max \left(t_{l}-s+1, e-t\right) \leq n c_{*}\right\}$, with $c_{*}$ as in Assumption II', there exists $1 \leq i^{*} \leq$ $i_{2}-i_{1}$ s.t. $\eta_{i_{1}+i^{*}} \in \mathcal{D}_{s, e}$ and $\left|\eta_{i_{1}+i^{*}}-b\right|<c_{0} \epsilon_{T}$ for $b=\operatorname{argmax}_{l \in \mathcal{D}_{s, e}}\left|\mathcal{C}_{s, l, e}\left(\widehat{z}_{k}\right)\right|$.

Proof The following covers the general discussion of the CUSUM statistic in the additive function plus noise setting. Using the simplified notation of this proof, if there is no change on $\left(u_{l-1}, u_{l}\right]$ we can write

$$
\widehat{z}_{k}\left(t_{l}\right)=z_{k}\left(t_{l}\right) Q_{l, k}=z_{k}\left(t_{l}\right)+z_{k}\left(t_{l}\right)\left(Q_{l, k}-1\right)
$$

where $Q_{l, k}$ is $\chi^{2}(1)$. The result for the case with a change point on $\left(u_{l-1}, u_{l}\right]$ follow directly by the arguments provided in the previous Lemma. This lets us formulate a generic model $\widehat{z}_{k}\left(t_{l}\right)=g\left(t_{l}\right)+e\left(t_{l}\right)$ with piecewise-constant function $g\left(t_{l}\right)$ (with possibly many change points). Following Venkatraman (1992, Lemmata 2.2-2.3), 
under Assumption II' and the conditions specified in Equations (8) and (9) for $g^{0}\left(t_{l}\right)$ denoting a one-step piecewise-constant function on $\{s, \ldots, e\}$ that has its change point $\eta$ on $\mathcal{D}_{s, e}$ and minimizes the mean-square distance between $g\left(t_{l}\right)$ and any onestep piecewise-constant function on $\{s, \ldots, e\}, \eta$ corresponds to one of the change points of $g\left(t_{l}\right)$ on $\{s, \ldots, e\}$.

In the framework of piecewise-constant function plus noise, detecting a change point on an interval $\{s, \ldots, e\}$ implies finding the mean-square optimal fit of a step function with a single step at $b$, denoted as $\widehat{g}_{b}\left(t_{l}\right)$. We know that for $b^{*}=$ $\operatorname{argmin}_{b} \sum_{l \in\{s, \ldots, e\}}\left(\widehat{z}_{k}\left(t_{l}\right)-\widehat{g}_{b}\left(t_{l}\right)\right)^{2}$,

$$
\sum_{l=s}^{e}\left(\widehat{z}_{k}\left(t_{l}\right)-g^{0}\left(t_{l}\right)\right)^{2}>\sum_{l=s}^{e}\left(\widehat{z}_{k}\left(t_{l}\right)-\widehat{g}_{b^{*}}\left(t_{l}\right)\right)^{2} .
$$

Therefore, if we can show that for some $c_{0} \epsilon_{T}$,

$$
\sum_{l=s}^{e}\left(\widehat{z}_{k}\left(t_{l}\right)-g^{0}\left(t_{l}\right)\right)^{2}<\sum_{l=s}^{e}\left(\widehat{z}_{k}\left(t_{l}\right)-\widehat{g}_{b}\left(t_{l}\right)\right)^{2}
$$

with $c_{0} \epsilon_{T}<|b-\eta|$, this would imply that $\left|b^{*}-\eta\right| \leq c_{0} \epsilon_{T}$. Subtracting the right part and expanding the above,

$$
\begin{aligned}
& \sum_{l=s}^{e}\left(g\left(t_{l}\right)+e\left(t_{l}\right)-g^{0}\left(t_{l}\right)\right)^{2}-\sum_{l=s}^{e}\left(g\left(t_{l}\right)+e\left(t_{l}\right)-\widehat{g}_{b}\left(t_{l}\right)\right)^{2} \\
= & \sum_{l=s}^{e}\left\{\left(g\left(t_{l}\right)-g^{0}\left(t_{l}\right)\right)^{2}-\left(g\left(t_{l}\right)-\widehat{g}_{b}\left(t_{l}\right)\right)^{2}\right\}+2 \sum_{l=s}^{e} e\left(t_{l}\right)\left(\widehat{g}_{b}\left(t_{l}\right)-g^{0}\left(t_{l}\right)\right) \equiv I+I I
\end{aligned}
$$

$I<0$ as $g^{0}\left(t_{l}\right)$ is defined to minimize the least squares fit to $g\left(t_{l}\right)$, over all step functions with a single step. We now show that $I$ absolutely exceeds $I I$ as $T \rightarrow \infty$. Let $\psi \in \Psi$ be those vectors defined on $\{s, \ldots, e\}$ with components that are first constant and positive and then constant and negative, such that these components are zero on average and squared sum up to one. Denoting as $\bar{g}$ the mean of $g\left(t_{l}\right)$ on $\{s, \ldots, e\}$ and $\psi^{0} \in \Psi$ s.t. $g^{0}\left(t_{l}\right)=\bar{g}+\left\langle g, \psi^{0}\right\rangle \psi^{0}\left(t_{l}\right)$ with $\langle\cdot\rangle$ denoting the inner 
product. Then

$$
\begin{aligned}
& \sum_{l=s}^{e}\left(g\left(t_{l}\right)-g^{0}\left(t_{l}\right)\right)^{2} \\
= & \sum_{l=s}^{e}\left(g\left(t_{l}\right)-\bar{g}\right)^{2}-2\left\langle g, \psi^{0}\right\rangle \sum_{l=s}^{e}\left(g\left(t_{l}\right)-\bar{g}\right) \psi^{0}\left(t_{l}\right)+\left\langle g, \psi^{0}\right\rangle^{2} \sum_{l=s}^{e}\left(\psi^{0}\left(t_{l}\right)\right)^{2} \\
= & \sum_{l=s}^{e}\left(g\left(t_{l}\right)-\bar{g}\right)^{2}-\left\langle g, \psi^{0}\right\rangle^{2}
\end{aligned}
$$

Let $\tilde{g}\left(t_{l}\right)$ be a step function that changes at $b$ and minimizes the squared distance to $g\left(t_{l}\right)$, then

$$
\sum_{l=s}^{e}\left(g\left(t_{l}\right)-\tilde{g}\left(t_{l}\right)\right)^{2} \leq \sum_{l=s}^{e}\left(g\left(t_{l}\right)+\widehat{g}_{b}\left(t_{l}\right)\right)^{2}
$$

Using Equations (13) and (16), for $\tilde{g}\left(t_{l}\right)=\bar{g}+\langle g, \tilde{\psi}\rangle \tilde{\psi}\left(t_{l}\right), I$ can be bounded as follows

$$
\begin{aligned}
& \sum_{l=s}^{e}\left\{\left(g\left(t_{l}\right)-g^{0}\left(t_{l}\right)\right)^{2}-\left(g\left(t_{l}\right)-\widehat{g}_{b}\left(t_{l}\right)\right)^{2}\right\} \\
\leq & \sum_{l=s}^{e}\left\{\left(g\left(t_{l}\right)-g^{0}\left(t_{l}\right)\right)^{2}-\left(g\left(t_{l}\right)-\tilde{g}\left(t_{l}\right)\right)^{2}\right\}=\left\langle g, \psi^{0}\right\rangle^{2}-\langle g, \tilde{\psi}\rangle^{2} \\
= & \left(\left|\left\langle g, \psi^{0}\right\rangle\right|-|\langle g, \tilde{\psi}\rangle|\right)\left(\left|\left\langle g, \psi^{0}\right\rangle\right|+|\langle g, \tilde{\psi}\rangle|\right) \leq\left(\left|\left\langle g, \psi^{0}\right\rangle\right|-|\langle g, \tilde{\psi}\rangle|\right)\left|\left\langle g, \psi^{0}\right\rangle\right|
\end{aligned}
$$

Note that $\left|\left\langle g, \psi^{0}\right\rangle\right|=\left|\mathcal{C}_{s, \eta, e}(g)\right|$ and $|\langle g, \tilde{\psi}\rangle|=\left|\mathcal{C}_{s, b, e}(g)\right|$. Thus, with the distance $c_{0} \epsilon_{T}$ between $\eta$ and $b$ the above is bounded by $-C \delta_{T}^{\prime 3} \epsilon_{T}$, by Lemmata 2 and 3 .

Consider now term $I I$ divided by 2 ,

$$
\begin{aligned}
& \sum_{l=s}^{e} e\left(t_{l}\right)\left(\widehat{g}_{b}\left(t_{l}\right)-g^{0}\left(t_{l}\right)\right) \\
= & \sum_{l=s}^{e} e\left(t_{l}\right)\left(\tilde{g}_{b}\left(t_{l}\right)-g^{0}\left(t_{l}\right)\right)+\sum_{l=s}^{e} e\left(t_{l}\right)\left(\widehat{g}_{b}\left(t_{l}\right)-\tilde{g}\left(t_{l}\right)\right)=I I . i+I I . i i
\end{aligned}
$$


For each of these sums we consider separately the following three constant intervals: assuming wlog $\eta<b, I I . i$ can be divided into

$$
\begin{aligned}
& \sum_{l=s}^{e} e\left(t_{l}\right)\left(\tilde{g}_{b}\left(t_{l}\right)-g^{0}\left(t_{l}\right)\right) \\
= & \sum_{l=s}^{\eta} e\left(t_{l}\right)\left(\tilde{g}_{b}\left(t_{l}\right)-g^{0}\left(t_{l}\right)\right)+\sum_{l=\eta+1}^{b} e\left(t_{l}\right)\left(\tilde{g}_{b}\left(t_{l}\right)-g^{0}\left(t_{l}\right)\right)+\sum_{l=b+1}^{e} e\left(t_{l}\right)\left(\tilde{g}_{b}\left(t_{l}\right)-g^{0}\left(t_{l}\right)\right) \\
= & I I . i .1+I I . i .2+I I . i .3
\end{aligned}
$$

By Lemmata 3 and 4 , as $T \rightarrow \infty$

$$
\begin{aligned}
|I I . i .1| & =\left|\frac{1}{\sqrt{\eta-s+1}} \sum_{l=s}^{\eta} e\left(t_{l}\right)\right|\left|\frac{\sqrt{\eta-s+1}}{b-s+1} \sum_{l=s}^{b} g\left(t_{l}\right)-\frac{1}{\sqrt{\eta-s+1}} \sum_{l=s}^{\eta} g\left(t_{l}\right)\right| \\
& \leq C \log T \frac{\sqrt{\eta-s+1} \sqrt{e-\eta}}{e-s+1} \epsilon_{T} /\left(\delta_{T}^{\prime} T\right) \mathcal{C}_{s, \eta, e}\left(z_{k}\right) \leq C^{\prime} \epsilon_{T} / \delta_{T}^{\prime} T \log T \mathcal{C}_{s, \eta, e}\left(z_{k}\right)
\end{aligned}
$$

Similar bounds can be derived for $\mid$ II.i.3 $\mid$ (of the same order as the above) and $\mid$ II.i.2 $\mid$ (of order $C^{\prime \prime} \sqrt{\epsilon_{T} /\left(\delta_{T}^{\prime} T\right)} \log T \mathcal{C}_{s, \eta, e}\left(z_{k}\right)$. Furthermore, II.ii can be decomposed as

$$
\begin{aligned}
\sum_{l=s}^{e} e\left(t_{l}\right)\left(\widehat{g}_{b}\left(t_{l}\right)-\tilde{g}\left(t_{l}\right)\right) & =\sum_{l=s}^{b} e\left(t_{l}\right)\left(\widehat{g}_{b}\left(t_{l}\right)-\tilde{g}\left(t_{l}\right)\right)+\sum_{l=b+1}^{e} e\left(t_{l}\right)\left(\widehat{g}_{b}\left(t_{l}\right)-\tilde{g}\left(t_{l}\right)\right) \\
& =\text { II.ii.1 }+ \text { II.ii.2 }
\end{aligned}
$$

II.ii.2 is of same order as II.ii.1, and, as $T \rightarrow \infty$,

$$
|I I . i i .1|=\frac{1}{b-s+1}\left(\sum_{l=1}^{b} e\left(t_{l}\right)\right)^{2}=\log ^{2} T
$$

Thus for $I$ to absolutely exceed $I I$ as $T \rightarrow \infty$, we require $\epsilon_{T} \delta_{T}^{\prime}>\max \left(\frac{\epsilon_{T} \log T}{\sqrt{T}}, \sqrt{\epsilon_{T} \delta_{T}^{\prime}} \log T, \log ^{2} T\right)$. This implies that $\epsilon_{T}>\log ^{2} T / \delta_{T}^{\prime 2}$, i.e. if $\epsilon_{T}=\max \left(t_{l}, \log ^{2+\vartheta} T\right)$ and $\vartheta>0$ suffice to meet the requirement derived above. Now we turn to the last Lemma needed to show consistent detection of change points 
for autospectra, before discussing the consistency of the stopping mechanism of the algorithm when there are no more change points to be detected. For the following, we require $\delta_{T}^{\prime-5 / 2} \log T<\sqrt{\epsilon_{T}}<\tau_{T}<\delta_{T}^{\prime} \sqrt{T}$, which implies $\tau_{T}=\kappa \log ^{1+\varpi} T$ with any $\varpi>\vartheta / 2$ and $\kappa>0$.

Lemma 6 Under the conditions stated in Equations (8) and (9) as $T \rightarrow \infty, \operatorname{Pr}(\mathcal{A}) \rightarrow$ 0 for $\mathcal{A}=\left\{\left|\mathcal{C}_{s, b, e}\left(\widehat{z}_{k}\right)\right|<\tau_{T} \sigma_{s, e}\left(\widehat{z}_{k}\right)\right\}$ with $b=\operatorname{argmax}_{l \in \mathcal{D}_{s, e}}\left|\mathcal{C}_{s, l, e}\left(\widehat{z}_{k}\right)\right|$.

Proof Define the event $\mathcal{B}_{f}=\left\{n^{-1}\left|\sum_{l=s}^{e} \widehat{z}_{k}\left(t_{l}\right)-\sum_{l=s}^{e} z_{k}\left(t_{l}\right)\right|<\breve{z}\right\}$ with $\breve{z} \equiv$ $(2 n)^{-1} \sum_{l=s}^{e} z_{k}\left(t_{l}\right)$. Using the Bernstein inequality (c.f. Lemma 4 ), it can be shown that $\operatorname{Pr}\left(\mathcal{B}_{f}\right) \rightarrow 1$ as $T \rightarrow \infty$, faster than for Equation (12) in Lemma 4. Thus $\operatorname{Pr}\left(n^{-1} \sum_{l=s}^{e} \widehat{z}_{k}\left(t_{l}\right) \in(\breve{z} / 2,3 \breve{z} / 2)\right) \rightarrow 1$ and, since $\operatorname{Pr}(\mathcal{A}) \leq \operatorname{Pr}\left(\mathcal{A} \cap \mathcal{B}_{f}\right)+\operatorname{Pr}\left(\mathcal{B}_{f}^{c}\right)$, it suffices to show that $\operatorname{Pr}\left(\mathcal{A} \cap \mathcal{B}_{f}\right) \rightarrow 0$. From Lemma 5, we know that there exists $1 \leq i^{*} \leq i_{2}-i_{1}$ s.t. $\eta_{i_{1}+i^{*}} \in \mathcal{D}_{s, e}$ and $\left|\eta_{i_{1}+i^{*}}-b\right|<c_{0} \epsilon_{T}$ for $b=\operatorname{argmax}_{l \in \mathcal{D}_{s, e}}\left|\mathcal{C}_{s, b, e}\left(\widehat{z}_{k}\right)\right|$. Wlog let $\eta_{i_{1}+i^{*}}<b$ and denote $z_{k, 1} \equiv z_{k}\left(\eta_{i_{1}+i^{*}}\right) \neq z_{k}\left(\eta_{i_{1}+i^{*}}+1\right) \equiv z_{k, 2}$. Then using Lemma 4 and Assumption II' in probability

$$
\begin{aligned}
\left|\mathcal{C}_{s, b, e}\left(\widehat{z}_{k}\right)\right| & \geq\left|\mathcal{C}_{s, b, e}\left(z_{k}\right)\right|-\log T \\
& \geq \sqrt{\frac{(b-s+1)(e-b)}{n}} \\
& \left|\frac{\left(\left(\eta_{i_{1}+i^{*}}-s+1\right) z_{k, 1}+\left(b-\left(\eta_{i_{1}+i^{*}}\right) z_{k, 2}\right.\right.}{b-s+1}-z_{k, 2}\right|-\log T \\
& \geq \sqrt{\frac{e-b}{n(b-s+1)}} z_{*}\left(\eta_{i_{1}+i^{*}}-s+1\right)-\log T \\
& \geq \sqrt{\frac{1-c_{*}}{n c_{*}}} z_{*}\left(\eta_{i_{1}+i^{*}}-s+1\right)-\log T \\
& \geq \frac{C \delta_{T}^{\prime} \sqrt{T}}{c^{*}}-\log T>\frac{3 \breve{z} \tau_{T}}{2}
\end{aligned}
$$

Lemma 7 For some $C, C^{\prime}>0$ let $\{s, e\}$ be such that either

1. $\exists 1 \leq k \leq N$ with $s \leq \eta_{i} \leq e$ and $\min \left(\eta_{i}-s+1, e-\eta_{i}\right) \leq C \epsilon_{T}$ or 


$$
\text { 2. } \exists 1 \leq k \leq N \text { with } s \leq \eta_{i}<\eta_{i+1} \leq e \text { and } \max \left(\eta_{i}-s+1, e-\eta_{+1 k}\right) \leq C^{\prime} \epsilon_{T}
$$

Then for $\mathcal{A}^{\prime}=\left\{\left|\mathcal{C}_{s, b, e}\left(\widehat{z}_{k}\right)\right|>\tau_{T} \sigma_{s, e}\left(\widehat{z}_{k}\left(t_{l}\right)\right)\right\}$ with $b=\operatorname{argmax}_{l \in \mathcal{D}_{s, e}}\left|\mathcal{C}_{s, l, e}\left(\widehat{z}_{k}\left(t_{l}\right)\right)\right|$, $\operatorname{Pr}\left(\mathcal{A}^{\prime}\right) \rightarrow 0$ as $T \rightarrow \infty$.

Proof We apply the same strategy as for the Proof of Lemma 6 using the event $\mathcal{B}_{f}$. We will show below that $\operatorname{Pr}\left(\mathcal{A} \cap \mathcal{B}_{f}\right) \rightarrow 0$. Assuming $\eta_{i}-s+1 \leq C \epsilon_{T}$ implies $b>\eta_{i}$, so

$$
\begin{aligned}
\left|\mathcal{C}_{s, b, e}\left(\widehat{z}_{k}\right)\right| & \leq\left|\mathcal{C}_{s, b, e}\left({ }^{-1} z_{k}\right)\right|+\log T \\
& \leq \sqrt{\frac{(b-s+1)(e-b)}{n}} \\
& \leq \sqrt{\frac{\left(\left(\eta_{i}-s+1\right) z_{k, 1}+\left(b-\left(\eta_{i}\right) z_{k, 2}\right.\right.}{b-s+1}-z_{k, 2} \mid+\log T} \\
& \leq \sqrt{\frac{e-\eta_{i}}{n\left(\eta_{i}-s+1\right)}} 2 z^{*}\left(\eta_{i}-s+1\right)+\log T \\
& \leq 2 z^{*} \sqrt{C \epsilon_{T}}+\log T<\frac{\breve{z} \tau_{T}}{2}
\end{aligned}
$$

The proof of the second case uses the same argument.

Functioning of the FreSpeD method for a single frequency band $\omega_{k}$ through Binary Segmentation The algorithm is initiated with $s=1, e=T$ and assuming there are $N>0$ sufficiently central change points (Assumption II') and the conditions of Equations 8 and 9 are met, Lemma 6 holds. Then the method detects a change point within the distance $c_{0} \epsilon_{T}$ from a true change point by Lemma 5 . The resulting two subsegments are such that Lemma 6 continues to hold. The algorithm continues until all change points are detected, and the segments resulting from the detected $N$ change points fulfill one of the criteria of Lemma 7 , so the algorithm terminates. 


\section{A.2 Thresholded CUSUM on the set of frequency bands}

$$
\omega_{k}, k=\left\{1, \ldots, K^{*}\right\}
$$

To ultimately prove Theorem 1 we need to show consistency of the thresholded sum of CUSUM statistics over all $K^{*}$ frequency bands. Let $\mathcal{K}=\left\{1, \ldots, K^{*}\right\}$ and $\mathcal{K}_{s, e} \subseteq \mathcal{K}$ the set of frequency bands with at least one change point in $z_{k}\left(t_{l}\right), l \in\{s, \ldots, e\}$, $t_{s}, t_{l}, t_{e} \in \mathbb{T}$. The following is based on the arguments in Cho and Fryzlewicz (2015a) on thresholded CUSUMs. By Lemma 4 we have $\max _{k} \max _{\{s, l, e\} \in \mathcal{D}}\left|\mathcal{C}_{s, l, e}\left(\widehat{z}_{k}\right)-\mathcal{C}_{s, l, e}\left(z_{k}\right)\right| \leq \log T$ with probability bounded from below by $1-\left(C K^{*} T^{3} \exp \left(-C \log ^{2} T\right)\right) \rightarrow 1$. Based on this event, on the one hand by Lemma 7 we know that the absolute CUSUMs of any $k \in \mathcal{K} \backslash \mathcal{K}_{s, e}$ will not exceed the threshold $\tau_{T}$ on the interval $\{s, \ldots, e\}$. On the other hand, by Lemma 6 the CUSUMs of frequency bands containing true change points will exceed the threshold around these change points with high probability.

The following is an observation on the behaviour of the CUSUMs of the true spectral quantities, which are piecewise-constant functions, based on Venkatraman (1992, Lemma 2.2). By Lemma 4 the CUSUMs of the estimated quantities are close. Con-

sider a generic piecewise-constant function $h_{k}(x)=\left(\alpha_{k, x} x+\beta_{k, x}\right) / \sqrt{(x(1-x))}$ with $x=\left(t_{l}-s+1\right) / n \in(0,1)$, where $\alpha_{k, x}, \beta_{k, x}$ depend on the change size and changepoint locations and are constant between change points. The scaling of the CUSUM by the locally estimated variability affects the magnitudes of these constants but does not affect the functional form of $h_{k}(x)$. These functions are either monotonic or v-shaped between two adjacent change points. They achieve their maximum over $\{s, \ldots, e\}$ at some change point contained in this interval.

The pointwise summation of the CUSUM statistics belonging to set $\mathcal{K}_{s, e}, h(x)$, has the same functional form as the individual components $h_{k}(x)$. We thus have on an 
interval $\{s, \ldots, e\}$,

$$
\begin{aligned}
\frac{\mathfrak{C}_{s, b, e}(\widehat{z})}{\left|\mathcal{K}_{s, e}\right|}= & \frac{\sum_{k=1}^{K^{*}} \mathcal{C}_{s, b, e}\left(\widehat{z}_{k}\right) / \sigma_{s, e}\left(\widehat{z_{k}}\right) \mathbb{I}\left(\mathcal{C}_{s, b, e}\left(\widehat{z}_{k}\right)>\tau_{T}\right)}{\left|\mathcal{K}_{s, e}\right|} \\
= & \frac{\sum_{k=1}^{K^{*}} \mathcal{C}_{s, b, e}\left(z_{k}\right) / \sigma_{s, e}\left(\widehat{z_{k}}\right) \mathbb{I}\left(\mathcal{C}_{s, b, e}\left(\widehat{z}_{k}\right)>\tau_{T}\right)}{\left|\mathcal{K}_{s, e}\right|} \\
& +\frac{\sum_{k=1}^{K^{*}}\left(\mathcal{C}_{s, b, e}\left(\widehat{z}_{k}\right)-\mathcal{C}_{s, b, e}\left(z_{k}\right)\right) / \sigma_{s, e}\left(z_{k}\right) \mathbb{I}\left(\mathcal{C}_{s, b, e}\left(\widehat{z}_{k}\right)>\tau_{T}\right)}{\left|\mathcal{K}_{s, e}\right|}
\end{aligned}
$$

where $\left|\mathcal{K}_{s, e}\right|$ denotes the cardinality of the set $\mathcal{K}_{s, e}$. By Lemma 4 the second part of the summation is bounded by $C \log T$. The first part of the summation can be seen as average of CUSUMs of piecewise-constant functions (from the set $\mathcal{K}_{s, e}$ ), rescaled by constants. Because thresholding has no effect in the neighbourhood $C \epsilon_{T}$ around a true change point, by Lemma $5 b=\operatorname{argmax}_{l \in\{s, e\}} \mathfrak{C}_{s, l, e}(\widehat{z})$ satisfies $\left|b-\eta_{i}\right|<c_{0} \epsilon_{T}$ for some $i$. The algorithm proceeds iteratively and detects all $N$ change points. It stops when all segments between change points satisfy the conditions of Lemma 7 for all $k=\left\{1, \ldots, K^{*}\right\}$.

We conclude with an outline of the consistency of our detection mechanism using the extra step of evaluating the thresholded CUSUM statistic on the interval $\mathcal{B}_{0}$ of length $\Delta_{T}+1$ around a change-point candidate $b_{0}$, which is shown in the pseudo-code representation of the FreSpeD method in Figure 3.2. Using the arguments of Lemma 3 , for any $k \in \mathcal{K}_{s, e}$ there exists $C>0$ s.t. $\mathcal{C}_{s, l, e}\left(z_{k}\right)>\tau_{T}$ for $\left|t-\eta_{i}\right|<C \epsilon_{T}$ for some change point $\eta_{i}$ of $z_{k}$ on $\{s, \ldots, e\}$. Then $\mathcal{C}_{s, b, e}\left(\widehat{z}_{k}\right)>\tau_{T}$ within a distance $\Delta_{T} \propto \epsilon_{T}$ for $b=\operatorname{argmax}_{l \in\{s, \ldots, e\}} \mathcal{C}_{s, l, e}\left(\widehat{z}_{k}\right)$ and thus this extra step is consistent. 


\section{SUPPLEMENTARY SECTION}

\section{B Simulation}

Simulation set up. To investigate the performance of the proposed methodology for finite samples, we conduct a simulation study using parametric models under the following settings:

- The total time series length is $T^{*}=50000$, in line with the EEG data analyzed in Section 4.

- The number of components is $D=2$ or 20 .

- The number of components that exhibit a change is denoted $D^{c}$. When $D=2$, we investigate the situation where $D^{c}=1$ or 2 ; when $D=20, D^{c}=1,10$ or 20 .

- The number of change points is $N=1$ or 5 , which are equidistant between themselves and to start and end of the time series.

A total of 500 multivariate time series were generated from each setting. In addition, we provide results for 100 simulation runs per setting for $D=128$ channels with 1, 64 or 128 components containing changes. While this setting is of interest in cognitive experimental settings, clinical applications typically have only about 20 channels (Trans-Cranial-Technologies, 2012).

We consider four parametric processes, $\operatorname{VAR}(2), \operatorname{VAR}(6), \operatorname{VAR}(10)$ and $\operatorname{VARMA}(2,2)$. Here, we only vary one or two parameters of the process components that undergo a change, letting them switch between parameter sets $A$ and $B$, while the remaining $D^{n c}=D-D^{c}$ components are fully described by parameter set $A$. The crosscorrelations of these processes are captured by the covariance matrix of the innovations. To illustrate, a general form of the $D$-variate $\operatorname{VAR}(p)$ process used here 
is

$$
\mathbf{X}\left(t^{*}\right)=\left[\begin{array}{c}
X_{1}\left(t^{*}\right) \\
X_{2}\left(t^{*}\right) \\
\cdots \\
X_{D}\left(t^{*}\right)
\end{array}\right]=\sum_{k=1}^{p}\left(\left[\begin{array}{cccc}
\phi_{k, 1} & 0 & \cdots & 0 \\
0 & \phi_{k, 2} & \cdots & 0 \\
\vdots & \vdots & \ddots & \vdots \\
0 & 0 & \cdots & \phi_{k, D}
\end{array}\right]\left[\begin{array}{c}
X_{1}\left(t^{*}-k\right) \\
X_{2}\left(t^{*}-k\right) \\
\cdots \\
X_{D}\left(t^{*}-k\right)
\end{array}\right]\right)+\left[\begin{array}{c}
\epsilon_{1}\left(t^{*}\right) \\
\epsilon_{2}\left(t^{*}\right) \\
\cdots \\
\epsilon_{D}\left(t^{*}\right)
\end{array}\right]
$$

where $\left[\epsilon_{1}\left(t^{*}\right), \ldots, \epsilon_{D}\left(t^{*}\right)\right]^{\prime}$ are i.i.d. Gaussian white noise with covariance $\Sigma$. Thus, we summarize the process parameter set by the vectors $\Phi_{k}=\left[\phi_{k, 1}, \ldots, \phi_{k, D}\right]^{\prime}$. The first $D^{c}$ elements of the parameter vector take the values $\phi_{k, j}=\phi_{k}^{A} \forall k \in\{1, \ldots, p\}$ before the first change point and $\phi_{k, j}=\phi_{k}^{B}$ afterwards, and alternate between these two values at each subsequent change point. The remaining $D^{n c}$ vector elements of $\Phi_{k}$ take the value $\phi_{k}^{A}$ throughout time. For the $\operatorname{VARMA}(2,2)$, the corresponding MA parameter vectors are denoted in this section $\Theta_{k}=\left[\theta_{k, 1}, \ldots, \theta_{k, D}\right]^{\prime}, k=\{1,2\}$ and their elements take the values $\theta_{k}^{A}$ or $\theta_{k}^{B}$ in the same manner as described for the VAR parameters above.

1. $\operatorname{VAR}(2):\left[\phi_{1}^{A}, \phi_{2}^{A}\right]^{\prime}=[-0.15,0.53]^{\prime},\left[\phi_{1}^{B}, \phi_{2}^{B}\right]^{\prime}=\left[-\phi_{1}^{A}, \phi_{2}^{A}\right]^{\prime}$

2. $\operatorname{VAR}(6):\left[\phi_{1}^{A}, \ldots, \phi_{6}^{A}\right]^{\prime}=[0.10,-0.10,0.00,0.10,0.20,-0.35]^{\prime}$ and $\left[\phi_{1}^{B}, \ldots, \phi_{6}^{B}\right]^{\prime}=\left[\phi_{1}^{A}, \ldots, \phi_{4}^{A},-\phi_{5}^{A},-\phi_{6}^{A}\right]^{\prime}$

3. $\operatorname{VAR}(10):\left[\phi_{1}^{A}, \ldots, \phi_{10}^{A}\right]^{\prime}=[0.10,-0.10,0.00,0.10,0.20,-0.35,0.00,0.00,0.20,-0.35]^{\prime}$ and $\left[\phi_{1}^{B}, \ldots, \phi_{10}^{B}\right]^{\prime}=\left[\phi_{1}^{A}, \ldots, \phi_{8}^{A},-\phi_{9}^{A},-\phi_{10}^{A}\right]^{\prime}$

4. $\operatorname{VARMA}(2,2):\left[\phi_{1}^{A}, \phi_{2}^{A}\right]^{\prime}=\left[\phi_{1}^{B}, \phi_{2}^{B}\right]^{\prime}=[-0.15,0.53]^{\prime},\left[\theta_{1}^{A}, \theta_{2}^{A}\right]^{\prime}=[0.10,-0.10]^{\prime}$ and $\left[\theta_{1}^{B}, \theta_{2}^{B}\right]^{\prime}=\left[2 \theta_{1}^{A}, 3 \theta_{2}^{A}\right]^{\prime}$

One realization of a changing component of each process with $N=5$ change points is displayed in Figure 7 and the autospectrum corresponding to a changing component of the $\operatorname{VAR}(6)$ process is given in Figure 8. The figures suggest that while it is difficult to visually spot the change points in the time series, these changes are more pronounced in the spectra, hereby illustrating the potential of using the spectral data features in a change-point detection algorithm for EEG data. 

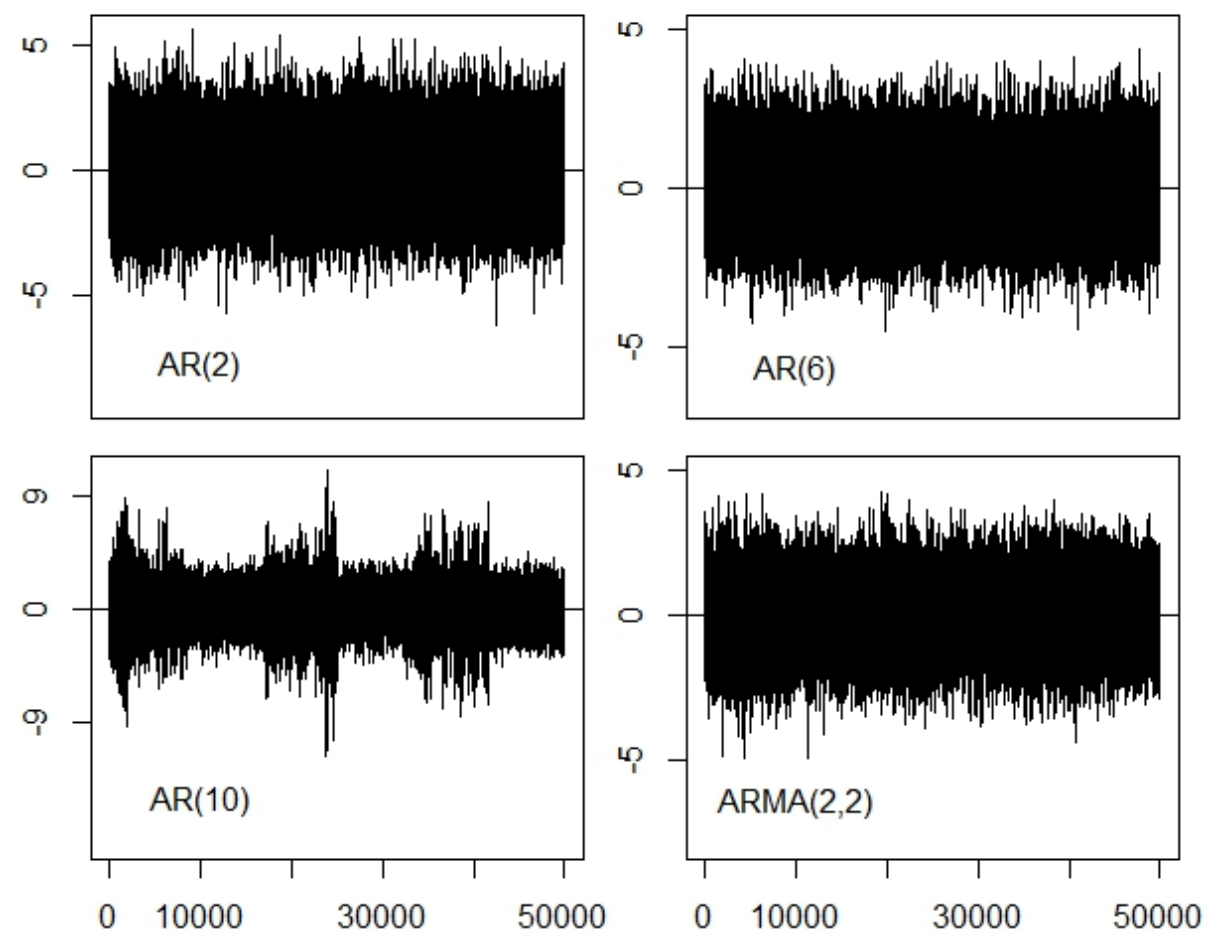

Figure 7: Realizations of the processes used in the simulation study, with a total of $N=5$ equidistant change points

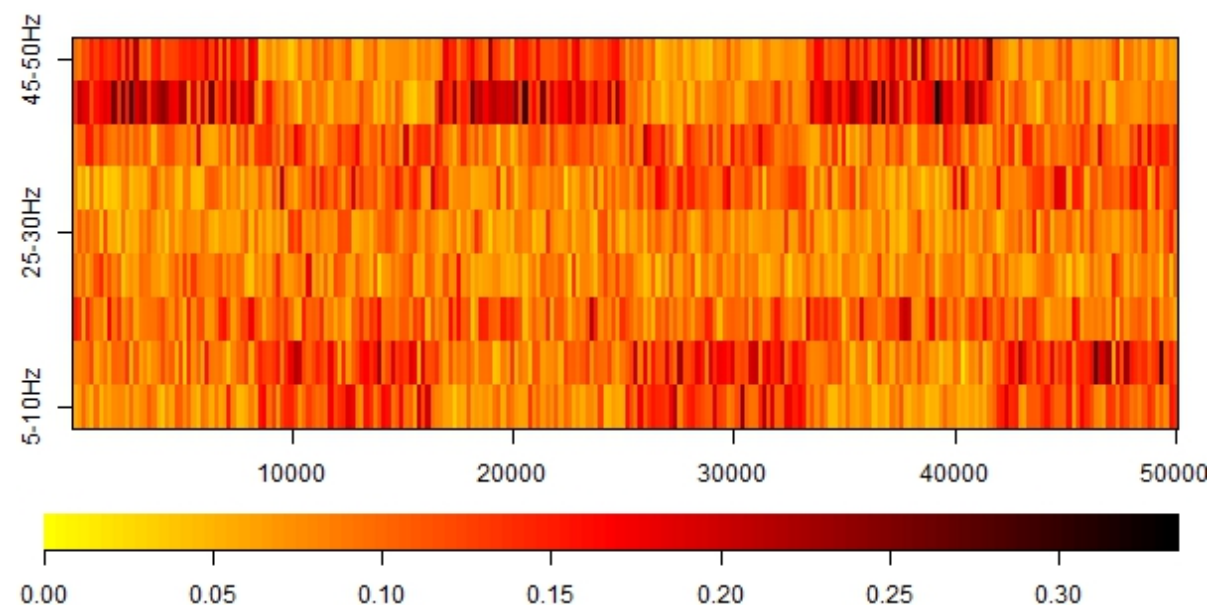

Figure 8: Estimated time-varying autospectrum of the VAR(2) processes depicted in Figure 7, assuming sampling rate of $100 \mathrm{~Hz}$ 
Remarks on competitor methods. The FreSpeD method is compared with approach of Cho and Fryzlewicz (2015a) using wavelet decompositions and the VARbased method of Kirch et al. (2015). Cho and Fryzlewicz (2015a) apply the principle of change-point detection via thresholded-sum CUSUM statistics to their multivariate Locally Stationary Wavelet model (LSW). In a similar way as FreSpeD analyzes estimates of spectral energy and coherence at individual frequency bands, LSW considers Haar wavelet periodograms and a transformation of the wavelet crossperiodograms at different scales. The LSW method is introduced together with practical guidance regarding the choice of parameters and the estimation of scale-specific thresholds. We use an implementation of the method and a bootstrap procedure for threshold estimation kindly provided by the authors, and the guidance provided in Cho and Fryzlewicz (2015a) for the choice of parameters.

The method of Kirch et al. (2015) (abbreviated KMO) is implemented with $p=6$. The choice of this order is somewhat arbitrary since KMO does not provide objective measures for selecting the optimal order for change-point estimation. KMO claims that the choice of the order should not adversely impact the result but the simulation studies suggest otherwise, with the most severe negative result occurring when the method uses an order that is smaller than that of the true underlying process. When KMO uses an order (here $p=6$ ) that is lower than that of the true process (e.g., $p=10$ ), it appears that one loses information in the dimension reduction step that could be crucial to change-point detection. In an unreported additional simulation study we found that KMO with order $p=2$ underperforms compared to the higher order parametrization $p=6$ and compared to FreSpeD.

The KMO method is not originally designed to identify more than two change points but, as stated in the paper, can be applied within a Binary Segmentation algorithm. The KMO method is a good benchmark to which FreSpeD can be compared because it is one of the very few methods that analyzes multi-channel EEG data of length exceeding a few thousand and at the same time provides flexible, nondyadic segmentation. While the spectral method in Preuß et al. (2015) is relevant and comparable to FreSpeD it is not feasible for analyzing big time series data such as the epileptic seizure data introduced in the following section, with $D=21$ and 
$T^{*}=50000$.

For a comparison of the methods with respect to computational speed, we conducted a brief comparison study on a 16-core AMD Opteron(TM) Processor 6276 with $2.3 \mathrm{GHz}$ and $252 \mathrm{~GB}$ RAM running Unix. For a fair comparison, we only consider processes where a method has identified the correct number of change points. The parametric KMO method is fastest with an average of 6 sec to identify a change point in a process with 20 channels. However, KMO requires the estimation of critical values which depend on $D$ and the interval length $e-s+1 \leq T^{*}$ and thus have to be estimated for a range of interval lengths within the Binary Segmentation framework. The calculation of a single threshold using code provided by the authors takes about $4.5 \mathrm{hr}$. In contrast, the FreSpeD method takes about $1.8 \mathrm{~min}$ but uses a single critical value that we discuss below. Moreover, FreSpeD can be parallelized, which scales down computational time by the order of $\mathrm{O}\left(D^{2}\right)$. The LSW method is less computationally attractive taking about twice as long as FreSpeD (3.8min) including the bootstrapping of threshold values.

In this numerical evaluation we chose as threshold for the FreSpeD method the value $\tau_{T}=0.8 \log ^{1.1}(T)$. The theoretical validity of this threshold is clear from Theorem 1 with the proof provided in the supplementary material. If this threshold is chosen too small, we risk detecting spurious change points. However, as the FreSpeD algorithm shows (see Figure 3.2), the step of testing the immediate neighbourhood of a change-point candidate generally reduces this risk. If we choose a too conservative threshold, we risk not detection true change points. This value provided good results in a small initial simulation for an $\mathrm{AR}(2)$ process with $T^{*}=50000, D=2, D^{c}=2$, $N=1, \nu=200$.

The evaluation of seizure data in the following section uses identical parameters. We provide simulation results for FreSpeD with varying window length $\nu \in$ $\{60,100,400,600\}$ at the end of this section; the results are similar to the ones for $\nu=200$, but as argued above the parametrization discussed in detail was chosen as it offers a good balance between frequency and time resolution for the data set with sampling rate $100 \mathrm{~Hz}$.

Further we set the scaled minimum change-point distance $\delta_{T}^{\prime} T=1400$ (14sec). 
Since both the KMO and the LSW method detect changes globally as opposed to at channel or channel-pair level, we adapt in this section a simple post-processing step to make the results from the FreSpeD method comparable. If the algorithm detects change points at two different channels or channel pairs that are very close to another (within a $\delta_{T}^{\prime} T$-distance) they are aggregated by averaging. 


\begin{tabular}{|c|c|c|c|c|c|c|c|c|c|c|c|c|c|}
\hline \multirow[t]{2}{*}{$D^{c}$} & \multirow[t]{2}{*}{ Process } & \multicolumn{4}{|c|}{ FreSpeD } & \multicolumn{4}{|c|}{ LSW } & \multicolumn{4}{|c|}{$\mathrm{KMO}, p=6$} \\
\hline & & $\hat{N}<N$ & $\hat{N}=N$ & $\hat{N}>N$ & MAD & $\hat{N}<N$ & $\hat{N}=N$ & $\hat{N}>N$ & MAD & $\hat{N}<N$ & $\hat{N}=N$ & $\hat{N}>N$ & MAD \\
\hline 1 & $\operatorname{VAR}(2)$ & 0.0 & 100.0 & 0.0 & 43.6 & 0.0 & 99.6 & 0.4 & 95.8 & 0.0 & 100.0 & 0.0 & 38.7 \\
\hline 1 & $\operatorname{VAR}(6)$ & 0.0 & 99.6 & 0.4 & 30.9 & 0.0 & 87.6 & 12.4 & 524.6 & 0.0 & 96.8 & 3.2 & 364.0 \\
\hline 1 & VAR(10) & 0.0 & 96.0 & 4.0 & 43.8 & 0.0 & 0.0 & 100.0 & NA & 0.0 & 44.4 & 55.6 & 99.1 \\
\hline 1 & $\operatorname{VARMA}(2,2)$ & 0.0 & 100.0 & 0.0 & 57.6 & 0.0 & 95.6 & 4.4 & 152.2 & 0.0 & 100.0 & 0.0 & 72.1 \\
\hline 2 & $\operatorname{VAR}(2)$ & 0.0 & 99.9 & 0.1 & 43.0 & 0.0 & 100.0 & 0.0 & 46.4 & 0.0 & 100.0 & 0.0 & 19.7 \\
\hline 2 & $\operatorname{VAR}(6)$ & 0.0 & 99.2 & 0.8 & 29.4 & 0.0 & 86.4 & 13.6 & 244.7 & 0.0 & 97.4 & 2.6 & 175.8 \\
\hline 2 & $\operatorname{VAR}(10)$ & 0.0 & 95.9 & 4.1 & 32.7 & 0.0 & 0.0 & 100.0 & NA & 0.0 & 47.6 & 52.4 & 41.3 \\
\hline 2 & $\operatorname{VARMA}(2,2)$ & 0.0 & 100.0 & 0.0 & 62.4 & 0.0 & 96.4 & 3.6 & 81.3 & 0.0 & 100.0 & 0.0 & 33.1 \\
\hline
\end{tabular}

Table 2: Single change point case for $D=2$. MAD: mean absolute distance between estimated and true change point for the results where $\hat{N}=N$.

\begin{tabular}{|c|c|c|c|c|c|c|c|c|c|c|c|c|c|}
\hline \multirow[t]{2}{*}{$D^{c}$} & \multirow[t]{2}{*}{ Process } & \multicolumn{4}{|c|}{ FreSpeD } & \multicolumn{4}{|c|}{ LSW } & \multicolumn{4}{|c|}{$\mathrm{KMO}, p=6$} \\
\hline & & $\hat{N}<N$ & $\hat{N}=N$ & $\hat{N}>N$ & MAD & $\hat{N}<N$ & $\hat{N}=N$ & $\hat{N}>N$ & MAD & $\hat{N}<N$ & $\hat{N}=N$ & $\hat{N}>N$ & MAD \\
\hline 1 & $\operatorname{VAR}(2)$ & 0.0 & 100.0 & 0.0 & 157.1 & 0.0 & 76.6 & 23.4 & 70.2 & 0.0 & 98.0 & 2.0 & 69.1 \\
\hline 1 & $\operatorname{VAR}(6)$ & 0.0 & 98.2 & 1.8 & 107.5 & 3.6 & 4.2 & 92.2 & 1701.8 & 90.6 & 9.0 & 0.4 & 467.7 \\
\hline تु & $\operatorname{VAR}(10)$ & 1.6 & 89.4 & 9.0 & 234.7 & 0.0 & 0.0 & 100.0 & NA & 14.8 & 7.0 & 78.2 & 3806.6 \\
\hline 1 & $\operatorname{VARMA}(2,2)$ & 62.2 & 37.8 & 0.0 & 433.6 & 0.0 & 50.0 & 50.0 & 91.2 & 0.0 & 94.8 & 5.2 & 103.9 \\
\hline 2 & $\operatorname{VAR}(2)$ & 0.0 & 100.0 & 0.0 & 159.2 & 0.0 & 78.4 & 21.6 & 38.3 & 0.0 & 98.8 & 1.2 & 37.4 \\
\hline 2 & $\operatorname{VAR}(6)$ & 0.0 & 98.7 & 1.3 & 116.1 & 0.0 & 4.6 & 95.4 & 225.1 & 6.6 & 68.4 & 25.0 & 252.9 \\
\hline 2 & $\operatorname{VAR}(10)$ & 1.9 & 88.6 & 9.5 & 234.8 & 0.0 & 0.0 & 100.0 & NA & 1.0 & 3.0 & 96.0 & 76.9 \\
\hline 2 & $\operatorname{VARMA}(2,2)$ & 61.4 & 38.6 & 0.0 & 432.1 & 0.0 & 50.2 & 49.8 & 47.6 & 0.0 & 96.8 & 3.2 & 55.7 \\
\hline
\end{tabular}

Table 3: Five change-points case for $D=2$. MAD: mean absolute distance between estimated and true change points for the results where $\hat{N}=N$. 


\begin{tabular}{|c|c|c|c|c|c|c|c|c|c|c|c|c|c|}
\hline \multirow[t]{2}{*}{$D^{c}$} & \multirow[t]{2}{*}{ Process } & \multicolumn{4}{|c|}{ FreSpeD } & \multicolumn{4}{|c|}{ LSW } & \multicolumn{4}{|c|}{$\mathrm{KMO}, p=6$} \\
\hline & & $\hat{N}<N$ & $\hat{N}=N$ & $\hat{N}>N$ & MAD & $\hat{N}<N$ & $\hat{N}=N$ & $\hat{N}>N$ & MAD & $\hat{N}<N$ & $\hat{N}=N$ & $\hat{N}>N$ & MAD \\
\hline 1 & $\operatorname{VAR}(2)$ & 0.0 & 99.8 & 0.2 & 39.3 & 0.0 & 87.0 & 13.0 & 92.1 & 0.0 & 100.0 & 0.0 & 41.1 \\
\hline 1 & $\operatorname{VAR}(6)$ & 0.0 & 98.6 & 1.4 & 34.9 & 0.0 & 3.6 & 96.4 & 176.5 & 30.8 & 69.2 & 0.0 & 440.5 \\
\hline 1 & $\operatorname{VAR}(10)$ & 0.0 & 96.6 & 3.4 & 45.5 & 0.0 & 0.0 & 100.0 & NA & 0.0 & 59.2 & 40.8 & 179.3 \\
\hline 1 & $\operatorname{VARMA}(2,2)$ & 0.0 & 100.0 & 0.0 & 82.4 & 0.0 & 10.3 & 89.7 & 52.0 & 0.0 & 100.0 & 0.0 & 72.1 \\
\hline 10 & $\operatorname{VAR}(2)$ & 0.0 & 99.9 & 0.1 & 33.7 & 0.0 & 91.4 & 8.6 & 9.5 & 0.0 & 100.0 & 0.0 & 6.5 \\
\hline 10 & $\operatorname{VAR}(6)$ & 0.0 & 99.4 & 0.6 & 42.4 & 0.0 & 7.0 & 93.0 & 24.3 & 0.0 & 100.0 & 0.0 & 41.7 \\
\hline 10 & $\operatorname{VAR}(10)$ & 0.0 & 95.5 & 4.5 & 29.7 & 0.0 & 0.0 & 100.0 & NA & 0.0 & 86.2 & 13.8 & 17.9 \\
\hline 10 & $\operatorname{VARMA}(2,2)$ & 0.0 & 100.0 & 0.0 & 69.7 & 0.0 & 16.0 & 84.0 & 8.2 & 0.0 & 100.0 & 0.0 & 8.9 \\
\hline 20 & $\operatorname{VAR}(2)$ & 0.0 & 99.9 & 0.1 & 31.8 & 0.0 & 91.6 & 8.4 & 5.0 & 0.0 & 100.0 & 0.0 & 5.7 \\
\hline 20 & $\operatorname{VAR}(6)$ & 0.0 & 99.3 & 0.7 & 40.2 & 0.0 & 6.7 & 93.3 & 1.7 & 0.0 & 100.0 & 0.0 & 21.4 \\
\hline 20 & $\operatorname{VAR}(10)$ & 0.0 & 95.7 & 4.3 & 31.2 & 0.0 & 0.0 & 100.0 & NA & 0.0 & 93.2 & 6.8 & 12.0 \\
\hline 20 & $\operatorname{VARMA}(2,2)$ & 0.0 & 100.0 & 0.0 & 71.2 & 0.0 & 21.0 & 79.0 & 2.8 & 0.0 & 100.0 & 0.0 & 6.9 \\
\hline
\end{tabular}

Table 4: Single change point case for $D=20$. MAD: mean absolute distance between estimated and true change point for the results where $\hat{N}=N$.

\begin{tabular}{|c|c|c|c|c|c|c|c|c|c|c|c|c|c|}
\hline \multirow[t]{2}{*}{$D^{c}$} & \multirow[t]{2}{*}{ Process } & \multicolumn{4}{|c|}{ FreSpeD } & \multicolumn{4}{|c|}{ LSW } & \multicolumn{4}{|c|}{$\mathrm{KMO}, p=6$} \\
\hline & & $\hat{N}<N$ & $\hat{N}=N$ & $\hat{N}>N$ & MAD & $\hat{N}<N$ & $\hat{N}=N$ & $\hat{N}>N$ & MAD & $\hat{N}<N$ & $\hat{N}=N$ & $\hat{N}>N$ & MAD \\
\hline 1 & $\operatorname{VAR}(2)$ & 0.0 & 99.6 & 0.4 & 158.8 & 10.0 & 83.0 & 7.0 & 874.2 & 67.0 & 33.0 & 0.0 & 85.8 \\
\hline 1 & $\operatorname{VAR}(6)$ & 0.0 & 98.4 & 1.6 & 115.1 & 20.0 & 9.0 & 71.0 & 3947.8 & 100.0 & 0.0 & 0.0 & NA \\
\hline 1 & $\operatorname{VAR}(10)$ & 0.6 & 90.4 & 9.0 & 235.9 & 0.0 & 0.0 & 100.0 & NA & 100.0 & 0.0 & 0.0 & NA \\
\hline 1 & $\operatorname{VARMA}(2,2)$ & 59.6 & 40.4 & 0.0 & 405.6 & 3.0 & 6.0 & 91.0 & 1079.0 & 100.0 & 0.0 & 0.0 & NA \\
\hline 10 & $\operatorname{VAR}(2)$ & 0.0 & 99.8 & 0.2 & 158.0 & 1.4 & 88.4 & 10.2 & 83.7 & 0.0 & 100.0 & 0.0 & 10.5 \\
\hline 10 & $\operatorname{VAR}(6)$ & 0.1 & 98.7 & 1.2 & 113.9 & 0.0 & 2.0 & 98.0 & 274.4 & 0.0 & 99.4 & 0.6 & 69.5 \\
\hline 10 & $\operatorname{VAR}(10)$ & 1.5 & 87.0 & 11.5 & 209.3 & 0.0 & 0.0 & 100.0 & NA & 0.0 & 21.2 & 78.8 & 40.1 \\
\hline 10 & $\operatorname{VARMA}(2,2)$ & 59.0 & 41.0 & 0.0 & 425.6 & 0.0 & 6.4 & 93.6 & 16.7 & 0.0 & 100.0 & 0.0 & 14.9 \\
\hline 20 & $\operatorname{VAR}(2)$ & 0.1 & 99.7 & 0.2 & 160.4 & 0.6 & 84.6 & 14.8 & 50.1 & 0.0 & 100.0 & 0.0 & 7.3 \\
\hline 20 & $\operatorname{VAR}(6)$ & 0.0 & 98.5 & 1.5 & 113.9 & 0.0 & 2.0 & 98.0 & 349.9 & 0.0 & 99.8 & 0.2 & 35.5 \\
\hline 20 & $\operatorname{VAR}(10)$ & 1.7 & 87.3 & 11.1 & 218.8 & 0.0 & 0.0 & 100.0 & NA & 0.0 & 22.6 & 77.4 & 30.1 \\
\hline 20 & $\operatorname{VARMA}(2,2)$ & 59.1 & 40.9 & 0.0 & 424.8 & 0.0 & 11.6 & 88.4 & 12.7 & 0.0 & 100.0 & 0.0 & 9.8 \\
\hline
\end{tabular}

Table 5: Five change-points case for $D=20$. MAD: mean absolute distance between estimated and true change points for the results where $\hat{N}=N$. 


\begin{tabular}{|c|c|c|c|c|c|c|c|c|c|c|c|c|c|}
\hline \multirow[t]{2}{*}{$D^{c}$} & \multirow[t]{2}{*}{ Process } & \multicolumn{4}{|c|}{ FreSpeD } & \multicolumn{4}{|c|}{ LSW } & \multicolumn{4}{|c|}{$\mathrm{KMO}, p=6$} \\
\hline & & $\hat{N}<N$ & $\hat{N}=N$ & $\hat{N}>N$ & MAD & $\hat{N}<N$ & $\hat{N}=N$ & $\hat{N}>N$ & MAD & $\hat{N}<N$ & $\hat{N}=N$ & $\hat{N}>N$ & MAD \\
\hline 1 & $\operatorname{VAR}(2)$ & 0.0 & 99.0 & 1.0 & 8.1 & 0.0 & 49.0 & 51.0 & $2,071.6$ & 0.0 & 100.0 & 0.0 & 55.5 \\
\hline 1 & $\operatorname{VAR}(6)$ & 0.0 & 97.0 & 3.0 & 24.7 & 0.0 & 0.0 & 100.0 & NA & 100.0 & 0.0 & 0.0 & NA \\
\hline 1 & $\operatorname{VAR}(10)$ & 0.0 & 78.0 & 22.0 & 2.6 & 0.0 & 0.0 & 100.0 & NA & 100.0 & 0.0 & 0.0 & NA \\
\hline 1 & $\operatorname{VARMA}(2,2)$ & 0.0 & 96.0 & 4.0 & 77.1 & 0.0 & 0.0 & 100.0 & NA & 22.0 & 78.0 & 0.0 & 81.3 \\
\hline 64 & $\operatorname{VAR}(2)$ & 0.0 & 99.0 & 1.0 & 15.6 & 0.0 & 49.0 & 51.0 & 2.4 & 0.0 & 100.0 & 0.0 & 5.5 \\
\hline 64 & $\operatorname{VAR}(6)$ & 0.0 & 97.0 & 3.0 & 8.7 & 0.0 & 0.0 & 100.0 & NA & 0.0 & 100.0 & 0.0 & 9.5 \\
\hline 64 & $\operatorname{VAR}(10)$ & 0.0 & 77.0 & 23.0 & 2.5 & 0.0 & 0.0 & 100.0 & NA & 0.0 & 100.0 & 0.0 & 9.3 \\
\hline 64 & $\operatorname{VARMA}(2,2)$ & 0.0 & 99.0 & 1.0 & 65.3 & 0.0 & 0.0 & 100.0 & NA & 0.0 & 100.0 & 0.0 & 6.1 \\
\hline 128 & $\operatorname{VAR}(2)$ & 0.0 & 99.0 & 1.0 & 15.8 & 0.0 & 54.0 & 46.0 & 1.3 & 0.0 & 100.0 & 0.0 & 5.8 \\
\hline 128 & $\operatorname{VAR}(6)$ & 0.0 & $97 / 0$ & 3.0 & 9.2 & 0.0 & 0.0 & 100.0 & NA & 0.0 & 100.0 & 0.0 & 6.5 \\
\hline 128 & $\operatorname{VAR}(10)$ & 0.0 & 77.0 & 23.0 & 2.9 & 0.0 & 0.0 & 100.0 & NA & 0.0 & 100.0 & 0.0 & 7.0 \\
\hline 128 & $\operatorname{VARMA}(2,2)$ & 0.0 & 99.0 & 1.0 & 62.8 & 0.0 & 0.0 & 100.0 & NA & 0.0 & 100.0 & 0.0 & 6.0 \\
\hline
\end{tabular}

Table 6: Single change point case for $D=128$. MAD: mean absolute distance between estimated and true change point for the results where $\hat{N}=N$. 


\begin{tabular}{|c|c|c|c|c|c|c|c|c|c|c|c|c|c|}
\hline \multirow[t]{2}{*}{$D^{c}$} & \multirow[t]{2}{*}{ Process } & \multicolumn{4}{|c|}{ FreSpeD } & \multicolumn{4}{|c|}{ LSW } & \multicolumn{4}{|c|}{$\mathrm{KMO}, p=6$} \\
\hline & & $\hat{N}<N$ & $\hat{N}=N$ & $\hat{N}>N$ & MAD & $\hat{N}<N$ & $\hat{N}=N$ & $\hat{N}>N$ & MAD & $\hat{N}<N$ & $\hat{N}=N$ & $\hat{N}>N$ & MAD \\
\hline 1 & $\operatorname{VAR}(2)$ & 0.0 & 80.0 & 20.0 & 146.1 & 41.0 & 16.0 & 43.0 & 697.0 & 100.0 & 0.0 & 0.0 & NA \\
\hline 1 & $\operatorname{VAR}(6)$ & 0.0 & 82.0 & 18.0 & 82.8 & 0.0 & 0.0 & 100.0 & NA & 100.0 & 0.0 & 0.0 & NA \\
\hline 1 & $\operatorname{VAR}(10)$ & 0.0 & 33.0 & 67.0 & 74.0 & 0.0 & 0.0 & 100.0 & NA & 100.0 & 0.0 & 0.0 & NA \\
\hline 1 & $\operatorname{VARMA}(2,2)$ & 42.0 & 27.0 & 31.0 & 374.8 & 0.0 & 0.0 & 100.0 & NA & 100.0 & 0.0 & 0.0 & NA \\
\hline 64 & $\operatorname{VAR}(2)$ & 0.0 & 84.4 & 15.6 & 131.8 & 1.0 & 21.0 & 78.0 & 11.9 & 0.0 & 100.0 & 0.0 & 5.9 \\
\hline 64 & $\operatorname{VAR}(6)$ & 0.0 & 82.1 & 17.9 & 82.8 & 0.0 & 0.0 & 100.0 & NA & 0.0 & 100.0 & 0.0 & 13.5 \\
\hline 64 & $\operatorname{VAR}(10)$ & 0.0 & 32.6 & 67.4 & 76.2 & 0.0 & 0.0 & 100.0 & NA & 0.0 & 64.0 & 36.0 & 26.9 \\
\hline 64 & $\operatorname{VARMA}(2,2)$ & 44.5 & 32.7 & 22.8 & 528.9 & 0.0 & 0.0 & 100.0 & NA & 0.0 & 100.0 & 0.0 & 7.1 \\
\hline 128 & $\operatorname{VAR}(2)$ & 0.0 & 83.8 & 16.2 & 131.5 & 0.0 & 33.0 & 67.0 & 3.4 & 0.0 & 100.0 & 0.0 & 5.6 \\
\hline 128 & $\operatorname{VAR}(6)$ & 0.0 & 82.5 & 17.5 & 82.9 & 0.0 & 0.0 & 100.0 & NA & 0.0 & 100.0 & 0.0 & 9.3 \\
\hline 128 & $\operatorname{VAR}(10)$ & 0.0 & 32.7 & 67.3 & 76.3 & 0.0 & 0.0 & 100.0 & NA & 0.0 & 51.0 & 49.0 & 22.0 \\
\hline 128 & $\operatorname{VARMA}(2,2)$ & 44.1 & 32.8 & 23.1 & 537.2 & 0.0 & 0.0 & 100.0 & NA & 0.0 & 100.0 & 0.0 & 7.1 \\
\hline
\end{tabular}

Table 7: Five change-points case for $D=128$. MAD: mean absolute distance between estimated and true change points for the results where $\hat{N}=N$. 
Discussion of results. The results of the simulation studies are reported in Tables 1 to 6 where the total number of change points are $N=1$ and $N=5$, respectively, and the number of components is $D=2, D=20$ and $D=128$. The columns denoted $\hat{N}<N, \hat{N}=N$ and $\hat{N}>N$ give the percentage among 500 simulated time series (100 for $D=128$ ) where the estimated number of change points is less than, equal to or larger than the true number of change points. The column MAD (mean absolute deviation) gives the average absolute distance between true and actual change points for those simulation runs where $\hat{N}=N$.

For the setting with only a single change point $(N=1)$ with a total of $D=2$ components and of which there is a change in only one $\left(D^{c}=1\right)$ or in both $\left(D^{c}=2\right)$ components: (a.) both KMO and FreSpeD perform well in all settings in terms of identifying the correct number of change points (from the column $\hat{N}=N$ ); (b.) LSW shows inferior performance with a tendency to overestimate $N$, and breaks down when the true process is $\operatorname{VAR}(10)$; (c.) FreSpeD beats KMO for the VAR(10) case - which again indicates that change-point detection in the reduced subspace can be problematic, especially when the changes are happening at VAR coefficient parameters at higher lags; (d.) FreSpeD identifies the location of the estimated change points to be closer to the true locations (as measured by the MAD) than LSW and KMO for most settings.

For the situation where $N=5$ change points and $D=2$ components, FreSpeD outperforms LSW and KMO in the three VAR processes in terms of estimating the correct number of change points. An exception is the $\operatorname{VARMA}(2,2)$ process where the change is subtle and takes place only in a very narrow frequency interval at the upper end of the frequency range. The FreSpeD method cannot isolate this change because, being a nonparametric approach, it attenuates this difference by smoothing within frequency bands which are broader than the narrow interval on which the difference exists. The LSW method performs slightly better than FreSpeD for the $\operatorname{ARMA}(2,2)$ case, but overestimates the number of change points when the process is AR with $p=6$ or $p=10$. While we can only speculate about the cause, it might be related to the way the threshold is simulated following Cho and Fryzlewicz (2015a), by bootstrapping under the assumption that the observed time series follows 
an $\mathrm{AR}(1)$ process. Moreover, conditional on correctly estimating the correct number of change points $N=5$, the mean absolute distance between true and estimated change points is overall larger for FreSpeD but, compared to KMO, displays a much less erratic behavior over processes. The accuracy of LSW is about the same order as $\mathrm{KMO}$ for $\operatorname{AR}(2)$ and $\operatorname{ARMA}(2,2)$.

Next, we examine the case where there are $D=20$ components and $N=1$ change points. Regardless of how many components $\left(D^{c}=1,10\right.$ or 20$)$ change, FreSpeD displays a stable behavior in terms of correctly identifying the number of change points. As the single change point is exactly on the border between two estimationinterval windows of length $\nu$, the accuracy is generally good (lower than $0.5 \nu$ ). In particular for $D^{c}=1 \mathrm{KMO}$ shows weaknesses as it tends to underestimate the number of change points in the $\operatorname{VAR}(6)$ case and tends to overestimate the number of change points in the $\operatorname{VAR}(10)$ case.

For the case $D=20$ components, $N=5$ change points, KMO beats FreSpeD when the true process is $\operatorname{VARMA}(2,2)$. A plausible explanation for the somewhat better performance of the KMO is that the $\operatorname{VARMA}(2,2)$ spectrum of the parametrization used here can be well approximated by a $\operatorname{VAR}(2)$ spectrum and, as discussed earlier, the change is only pronounced in a narrow frequency interval. However, for the same setting of many components and multiple change points, FreSpeD performs equally well or better than KMO when the true process is any of the three VAR processes. This is especially true for the most challenging case where only $D^{c}=1$ component (out of $D=20$ ) undergoes a change. Here, KMO has an undesirably high proportion of false negatives. This could have adverse consequences in monitoring EEGs of epilepsy patients because, as we demonstrate in the EEG analysis section, some changes are subtle and involve only a small subset of the channels. While FreSpeD was sensitive to these changes, it appears that the test statistic in the KMO method is dominated by the majority of the channels that exhibited no change. LSW shows a general tendency to overestimate the number of change points for the settings of $D=20$ and $N=\{1,5\}$, with exception of the $\operatorname{AR}(2)$ process.

Finally, for the case of $D=128$ which is more relevant to cognitive experiments rather than clinical settings (e.g., actual treatment of epilepsy), with a single change 
point $(N=1)$ FreSpeD is the only method with consistent performance estimating the correct number of change points for over $95 \%$ of simulation runs in all settings except for the $\operatorname{VAR}(10)$ cases, where this is the case in about three-quarters of time. KMO does not identify a change if it occurs in only one of 128 channels for $\operatorname{VAR}(6)$ and $\operatorname{VAR}(10)$ but otherwise is able to identify the number of changes correctly for all settings. LSW shows a clear tendency to overestimate the true number of change points. We find a similar picture for the case of $N=5$, where KMO underestimates the correct number of change points when the number of changing components is small, while FreSpeD overestimates the number of change points when the process is $\operatorname{VAR}(10)$ and does not identify the correct number of change points for the $\operatorname{VARMA}(2,2)$ process. The performance of FreSpeD appears to be generally independent of the number of channels with changes.

\section{Sensitivity to the Interval Length $\nu$}

We summarize the results of the simulation study specified in Section B below for varying interval length $\nu=\{60,100,400,600\}$. 


\begin{tabular}{|c|c|c|c|c|c|c|c|c|c|c|}
\hline \multirow[t]{2}{*}{$D$} & \multirow[t]{2}{*}{$D^{c}$} & \multirow[t]{2}{*}{ Process } & \multicolumn{4}{|c|}{$N=1, \nu=60$} & \multicolumn{4}{|c|}{$N=1, \nu=400$} \\
\hline & & & $\hat{N}<N$ & $\hat{N}=N$ & $\hat{N}>N$ & MAD & $\hat{N}<N$ & $\hat{N}=N$ & $\hat{N}>N$ & MAD \\
\hline 2 & 1 & $\mathrm{AR}(2)$ & 0.0 & 100.0 & 0.0 & 55.3 & 0.0 & 100.0 & 0.0 & 200.8 \\
\hline 2 & 1 & $\operatorname{AR}(6)$ & 0.0 & 100.0 & 0.0 & 42.4 & 0.0 & 99.8 & 0.2 & 200.0 \\
\hline 2 & 1 & $\operatorname{AR}(10)$ & 0.0 & 87.4 & 12.6 & 46.9 & 0.0 & 100.0 & 0.0 & 208.0 \\
\hline 2 & 1 & $\operatorname{ARMA}(2,2)$ & 0.0 & 100.0 & 0.0 & 111.4 & 0.0 & 100.0 & 0.0 & 226.4 \\
\hline 2 & 2 & $\operatorname{AR}(2)$ & 0.0 & 100.0 & 0.0 & 57.7 & 0.0 & 100.0 & 0.0 & 202.4 \\
\hline 2 & 2 & $\operatorname{AR}(6)$ & 0.0 & 100.0 & 0.0 & 41.5 & 0.0 & 99.9 & 0.1 & 202.8 \\
\hline 2 & 2 & $\mathrm{AR}(10)$ & 0.0 & 89.0 & 11.0 & 45.1 & 0.0 & 99.7 & 0.3 & 204.8 \\
\hline 2 & 2 & $\operatorname{ARMA}(2,2)$ & 0.0 & 100.0 & 0.0 & 101.0 & 0.0 & 100.0 & 0.0 & 228.4 \\
\hline \multirow[t]{2}{*}{$D$} & \multirow[t]{2}{*}{$D^{c}$} & \multirow[t]{2}{*}{ Process } & \multicolumn{4}{|c|}{$N=1, \nu=100$} & \multicolumn{4}{|c|}{$N=1, \nu=600$} \\
\hline & & & $\hat{N}<N$ & $\hat{N}=N$ & $\hat{N}>N$ & MAD & $\hat{N}<N$ & $\hat{N}=N$ & $\hat{N}>N$ & MAD \\
\hline 2 & 1 & $\operatorname{AR}(2)$ & 0.0 & 100.0 & 0.0 & 27.8 & 0.0 & 99.8 & 0.2 & 235.7 \\
\hline 2 & 1 & $\operatorname{AR}(6)$ & 0.0 & 100.0 & 0.0 & 15.8 & 0.0 & 100.0 & 0.0 & 216.0 \\
\hline 2 & 1 & $\mathrm{AR}(10)$ & 0.0 & 98.6 & 1.4 & 22.9 & 0.0 & 99.6 & 0.4 & 220.1 \\
\hline 2 & 1 & $\operatorname{ARMA}(2,2)$ & 0.0 & 100.0 & 0.0 & 91.6 & 0.0 & 100.0 & 0.0 & 260.8 \\
\hline 2 & 2 & $\operatorname{AR}(2)$ & 0.0 & 100.0 & 0.0 & 29.9 & 0.0 & 99.9 & 0.1 & 232.2 \\
\hline 2 & 2 & $\operatorname{AR}(6)$ & 0.0 & 100.0 & 0.0 & 14.3 & 0.0 & 100.0 & 0.0 & 219.6 \\
\hline 2 & 2 & $\mathrm{AR}(10)$ & 0.0 & 98.2 & 1.8 & 23.1 & 0.0 & 99.8 & 0.2 & 214.4 \\
\hline 2 & 2 & $\operatorname{ARMA}(2,2)$ & 0.0 & 100.0 & 0.0 & 77.9 & 0.0 & 100.0 & 0.0 & 254.8 \\
\hline
\end{tabular}

Table 8: Simulation results for varying interval length $\nu$ with $D=2$ components and $N=1$ change points. 


\begin{tabular}{|c|c|c|c|c|c|c|c|c|c|c|}
\hline \multirow[t]{2}{*}{$D$} & \multirow[t]{2}{*}{$D^{c}$} & \multirow[t]{2}{*}{ Process } & \multicolumn{4}{|c|}{$N=5, \nu=60$} & \multicolumn{4}{|c|}{$N=5, \nu=400$} \\
\hline & & & $\hat{N}<N$ & $\hat{N}=N$ & $\hat{N}>N$ & MAD & $\hat{N}<N$ & $\hat{N}=N$ & $\hat{N}>N$ & MAD \\
\hline 2 & 1 & $\operatorname{AR}(2)$ & 0.0 & 100.0 & 0.0 & 115.0 & 2.6 & 97.0 & 0.4 & 319.1 \\
\hline 2 & 1 & $\operatorname{AR}(6)$ & 0.0 & 99.6 & 0.4 & 70.6 & 1.2 & 98.6 & 0.2 & 266.9 \\
\hline 2 & 1 & $\mathrm{AR}(10)$ & 0.2 & 86.0 & 13.8 & 122.9 & 2.2 & 97.4 & 0.4 & 277.9 \\
\hline 2 & 1 & $\operatorname{ARMA}(2,2)$ & 22.8 & 77.2 & 0.0 & 290.9 & 81.6 & 18.4 & 0.0 & 495.9 \\
\hline 2 & 2 & $\operatorname{AR}(2)$ & 0.0 & 100.0 & 0.0 & 114.4 & 2.1 & 97.6 & 0.3 & 324.9 \\
\hline 2 & 2 & $\operatorname{AR}(6)$ & 0.0 & 99.8 & 0.2 & 70.8 & 1.9 & 97.9 & 0.2 & 277.6 \\
\hline 2 & 2 & $\mathrm{AR}(10)$ & 0.1 & 88.5 & 11.4 & 130.2 & 2.5 & 97.0 & 0.5 & 261.5 \\
\hline 2 & 2 & $\operatorname{ARMA}(2,2)$ & 23.6 & 76.4 & 0.0 & 289.6 & 80.2 & 19.8 & 0.0 & 510.7 \\
\hline \multirow[t]{2}{*}{$D$} & $\overline{D^{c}}$ & Process & \multicolumn{4}{|c|}{$N=5, \nu=100$} & \multicolumn{4}{|c|}{$N=5, \nu=600$} \\
\hline & & & $\hat{N}<N$ & $\hat{N}=N$ & $\hat{N}>N$ & MAD & $\hat{N}<N$ & $\hat{N}=N$ & $\hat{N}>N$ & MAD \\
\hline 2 & 1 & $\operatorname{AR}(2)$ & 0.0 & 99.8 & 0.2 & 110.0 & 26.4 & 73.6 & 0.0 & 474.8 \\
\hline 2 & 1 & $\operatorname{AR}(6)$ & 0.0 & 100.0 & 0.0 & 75.1 & 19.2 & 80.8 & 0.0 & 446.2 \\
\hline 2 & 1 & $\mathrm{AR}(10)$ & 0.2 & 96.0 & 3.8 & 129.6 & 8.4 & 91.6 & 0.0 & 387.8 \\
\hline 2 & 1 & $\operatorname{ARMA}(2,2)$ & 29.6 & 70.4 & 0.0 & 338.6 & 93.4 & 6.6 & 0.0 & 601.6 \\
\hline 2 & 2 & $\overline{A R}(2)$ & 0.0 & 99.7 & 0.3 & 117.5 & 25.2 & 74.8 & 0.0 & 484.2 \\
\hline 2 & 2 & $\operatorname{AR}(6)$ & 0.0 & 99.9 & 0.1 & 74.8 & 17.4 & 82.6 & 0.0 & 457.9 \\
\hline 2 & 2 & $\mathrm{AR}(10)$ & 0.1 & 95.3 & 4.6 & 131.3 & 9.4 & 90.5 & 0.1 & 378.0 \\
\hline 2 & 2 & $\operatorname{ARMA}(2,2)$ & 31.1 & 68.9 & 0.0 & 333.2 & 92.8 & 7.2 & 0.0 & 596.1 \\
\hline
\end{tabular}

Table 9: Simulation results for varying interval length $\nu$ with $D=2$ components and $N=5$ change points. 


\begin{tabular}{|c|c|c|c|c|c|c|c|c|c|c|}
\hline$D$ & $D^{c}$ & Process & & $N=1$, & $=60$ & & & $N=1, l$ & $=400$ & \\
\hline & & & $\hat{N}<N$ & $\hat{N}=N$ & $\hat{N}>N$ & MAD & $\hat{N}<N$ & $\hat{N}=N$ & $\hat{N}>N$ & MAD \\
\hline 20 & 1 & $\operatorname{AR}(2)$ & 0.0 & 100.0 & 0.0 & 55.8 & 0.0 & 99.8 & 0.2 & 201.6 \\
\hline 20 & 1 & $\operatorname{AR}(6)$ & 0.0 & 100.0 & 0.0 & 45.9 & 0.0 & 100.0 & 0.0 & 200.0 \\
\hline 20 & 1 & $\operatorname{AR}(10)$ & 0.0 & 89.6 & 10.4 & 46.7 & 0.0 & 100.0 & 0.0 & 200.8 \\
\hline 20 & 1 & $\operatorname{ARMA}(2,2)$ & 0.0 & 100.0 & 0.0 & 96.3 & 0.0 & 100.0 & 0.0 & 222.4 \\
\hline 20 & 10 & $\operatorname{AR}(2)$ & 0.0 & 100.0 & 0.0 & 58.3 & 0.0 & 100.0 & 0.0 & 203.8 \\
\hline 20 & 10 & $\operatorname{AR}(6)$ & 0.0 & 100.0 & 0.0 & 42.4 & 0.0 & 100.0 & 0.0 & 201.8 \\
\hline 20 & 10 & $\mathrm{AR}(10)$ & 0.0 & 89.1 & 10.9 & 45.6 & 0.0 & 99.9 & 0.1 & 201.4 \\
\hline 20 & 10 & $\operatorname{ARMA}(2,2)$ & 0.0 & 100.0 & 0.0 & 99.2 & 0.0 & 100.0 & 0.0 & 221.0 \\
\hline 20 & 20 & $\operatorname{AR}(2)$ & 0.0 & 100.0 & 0.0 & 56.8 & 0.0 & 100.0 & 0.0 & 204.2 \\
\hline 20 & 20 & $\operatorname{AR}(6)$ & 0.0 & 100.0 & 0.0 & 42.4 & 0.0 & 99.9 & 0.1 & 202.2 \\
\hline 20 & 20 & $\mathrm{AR}(10)$ & 0.0 & 89.2 & 10.8 & 45.4 & 0.0 & 99.9 & 0.1 & 202.2 \\
\hline 20 & 20 & $\operatorname{ARMA}(2,2)$ & 0.0 & 100.0 & 0.0 & 99.0 & 0.0 & 100.0 & 0.0 & 221.4 \\
\hline $\bar{D}$ & $D^{c}$ & Process & & $N=1, r$ & $=100$ & & & $N=1, l$ & $=600$ & \\
\hline & & & $\hat{N}<N$ & $\hat{N}=N$ & $\hat{N}>N$ & MAD & $\hat{N}<N$ & $\hat{N}=N$ & $\hat{N}>N$ & MAD \\
\hline 20 & 1 & $\operatorname{AR}(2)$ & 0.0 & 100.0 & 0.0 & 33.6 & 0.0 & 100.0 & 0.0 & 227.6 \\
\hline 20 & 1 & $\operatorname{AR}(6)$ & 0.0 & 100.0 & 0.0 & 16.2 & 0.0 & 99.8 & 0.2 & 219.2 \\
\hline 20 & 1 & $\mathrm{AR}(10)$ & 0.0 & 97.4 & 2.6 & 26.3 & 0.0 & 100.0 & 0.0 & 210.0 \\
\hline 20 & 1 & $\operatorname{ARMA}(2,2)$ & 0.0 & 100.0 & 0.0 & 73.2 & 0.0 & 100.0 & 0.0 & 250.8 \\
\hline 20 & 10 & $\mathrm{AR}(2)$ & 0.0 & 100.0 & 0.0 & 32.2 & 0.0 & 99.8 & 0.2 & 230.4 \\
\hline 20 & 10 & $\operatorname{AR}(6)$ & 0.0 & 100.0 & 0.0 & 15.9 & 0.0 & 99.9 & 0.1 & 228.4 \\
\hline 20 & 10 & $\operatorname{AR}(10)$ & 0.0 & 97.1 & 2.9 & 24.2 & 0.0 & 99.9 & 0.1 & 209.5 \\
\hline 20 & 10 & $\operatorname{ARMA}(2,2)$ & 0.0 & 100.0 & 0.0 & 75.1 & 0.0 & 100.0 & 0.0 & 254.3 \\
\hline 20 & 20 & $\operatorname{AR}(2)$ & 0.0 & 100.0 & 0.0 & 32.1 & 0.0 & 99.9 & 0.1 & 231.7 \\
\hline 20 & 20 & $\operatorname{AR}(6)$ & 0.0 & 100.0 & 0.0 & 15.5 & 0.0 & 99.9 & 0.1 & 225.6 \\
\hline 20 & 20 & $\mathrm{AR}(10)$ & 0.0 & 97.1 & 3.0 & 24.1 & 0.0 & 99.8 & 0.2 & 211.4 \\
\hline 20 & 20 & $\operatorname{ARMA}(2,2)$ & 0.0 & 100.0 & 0.0 & 75.2 & 0.0 & 100.0 & 0.0 & 253.6 \\
\hline
\end{tabular}

Table 10: Simulation results for varying interval length $\nu$ with $D=20$ components and $N=1$ change point. 


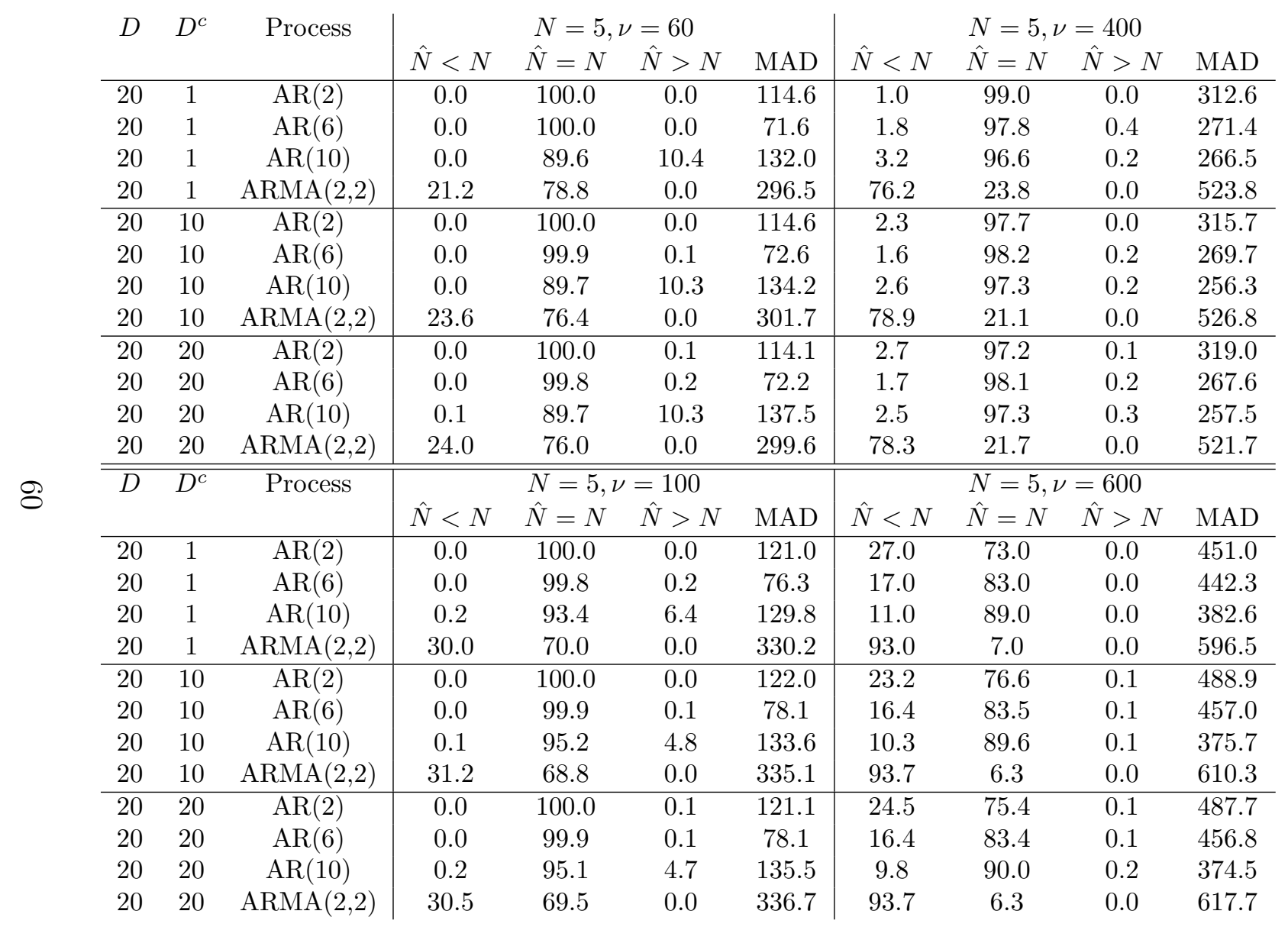

Table 11: Simulation results for varying interval length $\nu$ with $D=20$ components and $N=5$ change points. 